\title{
Low-fidelity covariances for neutron cross sections on 57 structural and 31 heavy nuclei in the fast region
}

\author{
M.T. Pigni ${ }^{\dagger}$, M. Herman, P. Obložinský \\ National Nuclear Data Center, Brookhaven National Laboratory \\ Upton, New York, 11973-5000, U.S.A. \\ www.nndc.bnl.gov
}

March 2008

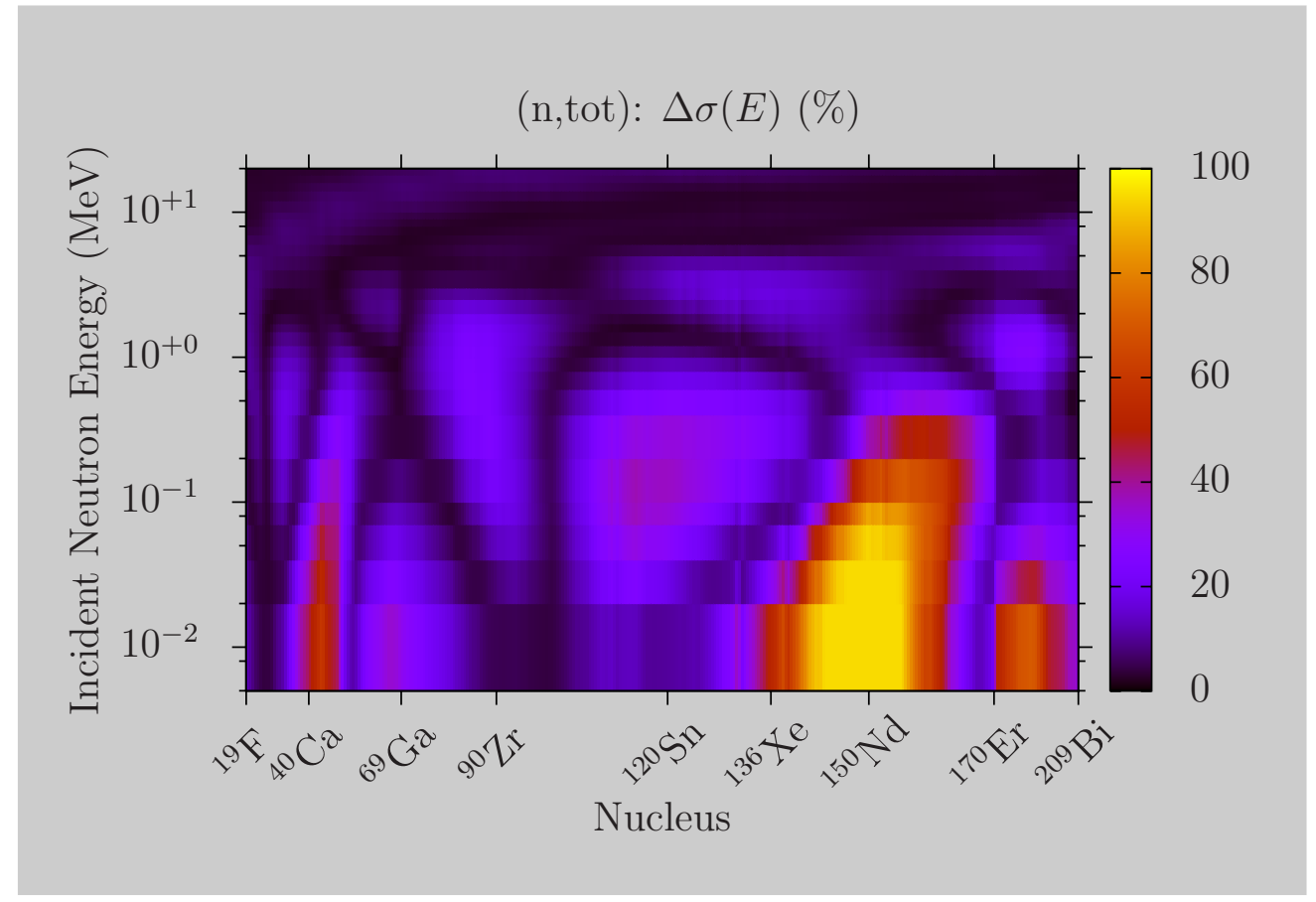

${ }^{\dagger}$ Reference Author: pigni@bnl.gov

Notice: This manuscript has been authored by employees of Brookhaven Science Associates, LLC under Contract No. DE-AC02-98CH10886 with the U.S. Department of Energy. The publisher by accepting the manuscript for publication acknowledges that the United States Government retains a non-exclusive, paid-up, irrevocable, world-wide license to publish or reproduce the published form of this manuscript, or allow others to do so, for United States Government purposes. 


\section{DISCLAIMER}

This report was prepared as an account of work sponsored by an agency of the United States Government. Neither the United States Government nor any agency thereof, nor any of their employees, nor any of their contractors, subcontractors, or their employees, makes any warranty, express or implied, or assumes any legal liability or responsibility for the accuracy, completeness, or any third party's use or the results of such use of any information, apparatus, product, or process disclosed, or represents that its use would not infringe privately owned rights. Reference herein to any specific commercial product, process, or service by trade name, trademark, manufacturer, or otherwise, does not necessarily constitute or imply its endorsement, recommendation, or favoring by the United States Government or any agency thereof or its contractors or subcontractors. The views and opinions of authors expressed herein do not necessarily state or reflect those of the United States Government or any agency thereof. 


\section{Contents}

1 Introduction 1

2 Evaluation Methodology 4

2.1 Evaluation method . . . . . . . . . . . . . . . . . 4

2.2 Reaction models and parameters . . . . . . . . . . . 6

2.2.1 Spherical optical model . . . . . . . . . . . . 7

2.2.2 Hauser-Feshbach statistical model . . . . . . . . . . . 8

2.2.3 Exciton pre-equilibrium model . . . . . . . . . . 9

2.2.4 Model parameters . . . . . . . . . . . . . . 9

2.3 Cross section sensitivities . . . . . . . . . . . . . . . 11

3 Calculations and Discussion 16

3.1 Calculations . . . . . . . . . . . . . . . 16

3.2 Discussion . . . . . . . . . . . . . . . . . . . . . . . . . . . . . . . . . . . . .

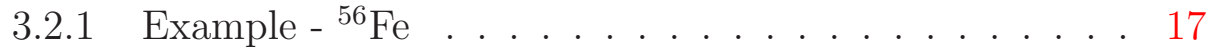

3.2.2 Materials from ${ }^{19} \mathrm{~F}$ to ${ }^{209} \mathrm{Bi}$. . . . . . . . . . . 27

4 Results 33

5 Conclusions and outlook $\quad 36$

$\begin{array}{ll}\text { List of Figures } & 39\end{array}$

$\begin{array}{ll}\text { List of Tables } & 40\end{array}$

$\begin{array}{ll}\text { Acknowledgments } & 41\end{array}$

$\begin{array}{ll}\text { Bibliography } & 44\end{array}$ 



\begin{abstract}
We produced a large set of neutron cross section covariances in the energy range of $5 \mathrm{keV}-20 \mathrm{MeV}$. The present set of data on 57 structural materials and 31 heavy nuclei follows our earlier work on 219 fission product materials and completes our extensive contribution to the low-fidelity covariance project (307 materials). This project aims to provide initial, low-fidelity yet consistent estimates of covariance data for nuclear criticality safety applications. The evaluation methodology combines the nuclear reaction model code EMPIRE which calculates sensitivity to nuclear reaction model parameters, and the Bayesian code KALMAN that propagates uncertainties of the model parameters to cross sections. Taking into account the large scale of the project, only marginal reference to experimental data was made. The covariances were derived from the perturbation of several key model parameters selected by the sensitivity analysis. These parameters refer to the optical model potential, the level densities and the strength of the pre-equilibrium emission. This work represents the first attempt ever to generate nuclear data covariances on such a large scale.
\end{abstract}





\section{Chapter 1}

\section{Introduction}

Recent revival of activities in neutron cross section covariances can be explained by two factors. First, there is intensive renewed interest in nuclear technology applications, such as the design of new generation of nuclear power reactors and enhanced requirements on nuclear criticality safety. Second, considerable advances in computer technology and improvements in neutronics simulation codes allow refined prediction of integral quantities that start to probe the uncertainties of basic nuclear data.

Neutron cross section covariances (uncertainties and correlations) are needed for several distinct types of applications. Probably the most important among them is the need to assess uncertainties of integral quantities such as design and operational parameters of nuclear power reactors. An estimation of the accuracy of predictions of such applied quantities due to the uncertainties of the basic data can be viewed as forward propagation of uncertainties. To this end one computes sensitivity coefficients for the integral quantity $R$ with respect to cross sections and obtains the integral variance as

$$
(\Delta R)^{2}=\mathbf{s}_{R}^{\mathrm{T}} \mathbf{D} \mathbf{s}_{R}
$$

where $\mathbf{s}_{R}^{\mathrm{T}}$ is the transpose of the sensitivity coefficient vector $\mathbf{s}_{R}$ and $\mathbf{D}$ is the cross section covariance matrix.

The same approach, albeit in the reverse order, can be used to identify nuclear data needs. In doing so one starts from the target uncertainties of integral quantities and propagates them backwards to the desired precision of neutron cross sections. Recent international effort by Salvatores et al. [1] applied this approach to identify the data needs for advanced reactor systems. 
Data adjustment represents another important use of neutron covariance data. To this end, one would start with the basic evaluated data library, such as the recently released ENDF/B-VII.0 [2], analyze suitable integral experiments, calculate sensitivities for observed integral quantities, account for covariance data, and produce an adjusted multi-group library constrained by these integral experiments. Such an adjusted library should then be used for neutronics simulations of the new systems. Ideally, if adjustments were propagated backward to the basic library, one would also be able predict integral quantities for systems that go beyond the current experience in terms of the composition of materials and neutron energy spectra. The Global Nuclear Energy Partnership, GNEP, is currently engaged in such a data adjustment project, focusing on fast, metal-cooled actinide burner reactors [3].

Although covariances were extensively studied in the 1970's, this earlier activity virtually disappeared in the 1990's due to limited interest by users and scarce resources for data evaluation. As a consequence, the availability of covariances in the major nuclear data libraries is very limited. For example, the most recent ENDF/B-VII.0 library, released in December 2006, contains covariances for 26 materials only, i.e., for less than $7 \%$ of the materials included in the neutron sub-library. Moreover, only 13 of these materials provide covariances for all reaction channels important for applications and can, therefore, be considered complete. The lack of a consistent and complete set of covariances is a barrier that prevents using the sensitivity tools in the development of innovative nuclear technologies and discourages the advancement of the tools themselves.

The issue of scarce neutron covariance data was recently addressed by the U.S. nuclear criticality safety program. In order to meet nuclear criticality safety requirements and allow for testing and further development of their computational tools, the "low-fidelity" covariance project for nuclear criticality safety has been initiated. Even relatively crude approximations would be an enormous help for these developments. The goal of this project is to produce rough set of covariances covering all relevant reaction channels and materials (from the thermal energy to $20 \mathrm{MeV}$ ) and provide a solid base for testing the new tools for advanced numerical simulations employing nuclear data uncertainties and correlations. The emphasis is on completeness rather than on precision - the latter should be addressed later once the evaluation methodology is well established and adequate tools are available.

In view of the large scale of the low-fidelity covariance project, our results are based on model calculations with minimal reference to experimental data. 
Calculations by the BNL code EMPIRE [4] with default set of parameters provide a complete set of cross sections, while the LANL code KALMAN [5] generates their (co)variances. We stress that EMPIRE predictions of cross sections, although very reasonable in many instances, do not coincide with any of the official evaluated libraries. Therefore, our covariances should not be associated with any cross sections recommended for applications. An almost complete neglect of experimental data, and the global nature of our model calculations also limits detailed comparison with the existing covariances that result from a much more thorough analysis.

The aim of the low-fidelity neutron cross section covariance project is to produce cross section covariances (MF33 in ENDF-6 format definition) for a complete set of materials included in the ENDF/B-VII.0 neutron sublibrary. The data should cover five major reaction channels, (n,el), (n,inl), $(n, 2 n),(n, \gamma)$ and $(n, f)$, with the understanding that $(n, t o t)$ is redundant. The project involves four national laboratories with the following responsibilities:

- ORNL - Low neutron energy region $(<5 \mathrm{keV})$ for all nuclei

- BNL - Fast neutron energy region (5 keV - $20 \mathrm{MeV}$ ) for nuclei $19 \leq \mathrm{A} \leq 209$

- LANL - Fast neutron energy region for light nuclei, $\mathrm{A} \leq 18$, and for all actinides, $\mathrm{A} \geq 210$

- ANL - Checking and reviewing

Our role was to produce covariances in the fast neutron region for 307 materials, ${ }^{19} \mathrm{~F}-{ }^{209} \mathrm{Bi}$. This massive task was split into two parts. First, we produced covariances for 219 fission products [6] defined as the ENDF/BVII.0 materials in the range of $\mathrm{Z}=31-68$ and presented our results at the recent international conference [7] as well as the CSEWG'2007 meeting. Second, we produced covariances for the remaining 57 structural materials and 31 heavy materials and these are reported in the present document.

The report is organized as follows. In Chapter 2, we describe the methodology, while the calculations are presented and discussed in Chapter 3. The results are summarized in Chapter 4 and followed by our conclusions in Chapter 5 . 


\section{Chapter 2}

\section{Evaluation Methodology}

Our methodology is based on the BNL nuclear reaction model code EMPIRE [4] coupled to the LANL Bayesian filtering code KALMAN [5]. For the purposes of this project the results are almost entirely based on model calculations with marginal reference to experimental data. The EMPIRE code calculates cross sections according to appropriately selected models and model parameters, while KALMAN propagates the model parameter uncertainties into cross section (co)variances.

\subsection{Evaluation method}

The Bayesian update procedure is a standard tool used in statistics to modify prior by taking into account the new data. In our case, the evaluation starts with the EMPIRE nuclear reaction model code which makes use of a wide range of nuclear models of different degrees of sophistication in order to provide an overall description of nuclear observables. The adopted models address specific reaction mechanisms and depend on adjustable parameters. The most relevant model parameters are those related to optical potential and nuclear level densities. These parameters are varied to calculate partial derivatives of cross sections, defining the elements of the sensitivity matrices (e.g., Eq. (2.5)). The calculated reaction cross sections, sensitivity matrices along with the model parameters and their uncertainties represent input quantities for the KALMAN code.

In general, the code KALMAN is used as a nuclear data evaluation tool based on the iterative least-squares approach. The procedure puts emphasis on the estimation of the model parameter uncertainties and the corresponding 
correlations. It is applied to the evaluation of neutron cross sections and their covariance matrices for various reaction channels. The application of the Bayesian equations is straightforward and the update is a simple algebraic operation,

$$
\begin{gathered}
\mathbf{p}_{n+1}=\mathbf{p}_{n}+\mathbf{P}_{n} \mathbf{S}^{\mathrm{T}} \mathbf{Q}_{n}\left(\boldsymbol{\sigma}_{n}^{\exp }-\boldsymbol{\sigma}\left(\mathbf{p}_{n}\right)\right) \\
\mathbf{P}_{n+1}=\mathbf{P}_{n}-\mathbf{P}_{n} \mathbf{S}^{\mathrm{T}} \mathbf{Q}_{n} \mathbf{S} \mathbf{P}_{n}
\end{gathered}
$$

where $n$ denotes the $n^{\text {th }}$-step in the evaluation process according to the number of sets of experimental data to be included. The vector $\mathbf{p}_{n+1}$ contains the improved values of the parameters starting from the vector $\mathbf{p}_{n}$. Likewise, the matrix $\mathbf{P}_{n+1}$ is the updated covariance matrix of the parameters $\mathbf{p}_{n+1}$. The combination of the theoretical covariance matrix $\mathbf{D}$ and the experimental covariances matrix $\mathbf{D}^{\exp }$ results in the updated covariance matrix,

$$
\mathbf{Q}=\left(\mathbf{D}^{\exp }+\mathbf{D}\right)^{-1}
$$

and the vector $\boldsymbol{\sigma}(\mathbf{p})$ represents the set of cross sections for a specific reaction channel calculated for the set of parameters $\mathbf{p}$.

In the present project the experimental data were virtually ignored, considerably simplifying the evaluation procedure that become driven by the theoretical cross section covariances. The theoretical cross section covariance matrix,

$$
\mathrm{D}=\mathrm{SPS}^{\mathrm{T}}
$$

is associated with the model calculation by the correlation matrix of the model parameters,

$$
\mathbf{P} \equiv\left\langle\Delta p_{\ell} \Delta p_{m}\right\rangle
$$

and the sensitivity matrix $\mathbf{S}$ with the elements,

$$
s_{i, j}=\frac{\partial \sigma\left(E_{i}, \mathbf{p}\right)}{\partial p_{j}},
$$

calculated as the partial derivative of the cross section $\sigma$ at the energy $E_{i}$ with respect to the parameter $p_{j}$. Then, the low-fidelity covariance matrix can be readily identified with the theoretical covariance matrix,

$$
\mathrm{D}_{\text {low-fi }}=\mathbf{D} \text {. }
$$

In the explicit notation, the cross section covariance matrix elements can be written as

$$
d_{i, k}=\sum_{\ell, m=1}^{q} s_{i, \ell}\left\langle\Delta p_{\ell} \Delta p_{m}\right\rangle s_{i, m}
$$


Here, the covariance matrix of model parameters, $\mathbf{P}$, is diagonal, if model parameters are uncorrelated as assumed in the present work. Conveniently, the cross section covariance matrix can be separated into the diagonal terms - cross section uncertainties and the cross section correlation matrix defined as

$$
\zeta_{i, k}=\frac{d_{i, k}}{\sqrt{d_{i, i}} \sqrt{d_{k, k}}},
$$

where the matrix elements are normalized with the values in the range of $-1 \leq \zeta_{i, j} \leq 1$.

It is important to note that the covariance matrix must be symmetric and definite positive. While the first requirement is easy to satisfy, the second is less transparent and it means that

$$
\mathbf{Z D Z}^{\mathrm{T}}>0,
$$

for all non-zero real vectors $\mathbf{Z}$. This is achieved by making sure that after diagonalizing all matrix elements have positive values. The point is that numerical rounding errors in the calculation and normalization procedure can lead to inconsistencies, with the matrices $\boldsymbol{\zeta}$ not satisfying Eq. (2.9). In the present work, all cross section covariance matrices were carefully checked to ensure definite-positiveness.

\subsection{Reaction models and parameters}

The EMPIRE code system [4] is a modern tool for modeling low and intermediate energy nuclear reactions. It incorporates an extensive set of nuclear reaction models capable of describing all relevant reaction mechanisms, each of them conveniently coupled to the up-to-date library of input model parameters [8]. Therefore, EMPIRE provides reasonable overall description of nuclear observables even if default parametrization is being used. The code is suitable for massive calculations, is easy to use, has readily available default input values for all parameters, and it is applicable to wide range of target nuclei and incident neutron energies from about $1 \mathrm{keV}$ to $150 \mathrm{MeV}$.

For the purposes of the present project, three nuclear reaction models were adopted:

- spherical optical model, 
- Hauser-Feshbach statistical model,

- exciton pre-equilibrium model.

These three models should sufficiently well describe the physics of nuclear reactions at neutron energies from $5 \mathrm{keV}$ to $20 \mathrm{MeV}$ for both the structural materials and heavy nuclei. The spherical optical model takes care of the total cross sections and neutron elastic scattering, the Hauser-Feshbach statistical model describes the bulk of particle emission, and the exciton preequilibrium model describes major features of fast particle emission at higher incident energies.

For better understanding of the model parameters considered in our calculations, we give a brief and simplified description of the nuclear reaction models mentioned above. This will be followed by discussion of model parameters and their uncertainties.

\subsubsection{Spherical optical model}

The optical model for nucleon-nucleus interaction is the starting ingredient in cross section calculations. This model allows to calculate neutron elastic scattering as well as absorption cross sections and transmission coefficients discussed later. The spherical optical model potential is usually defined as

$\mathcal{U}(r, E)=-\mathcal{V}_{v}(r, E)-i \mathcal{W}_{v}(r, E)-i \mathcal{W}_{s}(r, E)+\mathcal{V}_{s o}(r, E) \ell \cdot s+i \mathcal{W}_{s o}(r, E) \ell \cdot s$.

Here, all components are separated in E-dependent well depths and energyindependent radial parts according to

$$
\begin{aligned}
\mathcal{V}_{v} & =V_{v}(E) f\left(r, R_{v}, a_{v}\right) \\
\mathcal{W}_{v} & =W_{v}(E) f\left(r, R_{v}, a_{v}\right) \\
\mathcal{W}_{s} & =-4 a_{s} W_{s}(E) \frac{\mathrm{d}}{\mathrm{d} r} f\left(r, R_{s}, a_{s}\right) \\
\mathcal{V}_{s o} & =V_{s o}(E)\left(\frac{\hbar}{m_{\pi} c}\right)^{2} \frac{1}{r} \frac{\mathrm{d}}{\mathrm{d} r} f\left(r, R_{s o}, a_{s o}\right) \\
\mathcal{W}_{s o} & =W_{s o}(E)\left(\frac{\hbar}{m_{\pi} c}\right)^{2} \frac{1}{r} \frac{\mathrm{d}}{\mathrm{d} r} f\left(r, R_{s o}, a_{s o}\right)
\end{aligned}
$$


where the indices $v, s$ and so refer to volume-central, surface-central and spin-orbit potentials, respectively. The form-factor is given by the frequently used Woods-Saxon shape

$$
f\left(r, R_{i}, a_{i}\right)=\left(1+\exp \left[\left(r-R_{i}\right) / a_{i}\right]\right)^{-1},
$$

where the geometry parameters are the radius $R_{i}=r_{i} A^{1 / 3}$ and the diffuseness $a_{i}$, with $A$ being the atomic mass number.

\subsubsection{Hauser-Feshbach statistical model}

The decay of the compound nucleus is described in the framework of the Hauser-Feshbach statistical model formalism with many open channels contributing to the decay. Schematically, the cross section for a reaction $(a, b)$ that proceeds through the compound nucleus $(\mathrm{CN})$ mechanism can be written as

$$
\sigma_{a, b}=\sigma_{a} \frac{\Gamma_{b}}{\sum_{c} \Gamma_{c}} .
$$

Here, $\sigma_{a}$ is the absorption cross-section and the ratio expresses the chance of emitting particle $b$ relative to all other reaction channels. The notation showing summation over the quantum numbers such as spin and parity, and integration over the energy is suppressed for simplicity. The decay width is given by

$$
\Gamma_{c}=\int_{0}^{E-B_{c}} \frac{\rho_{c}\left(E^{\prime}\right) T_{c}\left(E-B_{c}-E^{\prime}\right) \mathrm{d} E^{\prime}}{2 \pi \rho_{\mathrm{CN}}(E)},
$$

where $B_{c}$ is the binding energy of particle $c$ in the compound nucleus, $\rho$ is the nuclear level density, and $T_{c}(\epsilon)$ stands for the transmission coefficient for particle $c$ having channel energy $\epsilon=E-B_{c}-E^{\prime}$. Again, we dropped explicit reference to the spin and parity. Since all evaluations extend up to $20 \mathrm{MeV}$, sequential multi-particle particle emission had to be included in the Hauser-Feshbach calculations, which in practice implies an energy convolution of multiple integrals of the type of Eq. (2.14).

EMPIRE offers several models describing nuclear level densities. We adopted the EMPIRE-specific approach [4] that uses the formalism of the super-fluid model below the critical excitation energy and the Fermi gas model above this energy. The dominant term is

$$
\rho(E) \propto a^{1 / 4} \exp (2 \sqrt{\text { const } \cdot a}),
$$

where the level density parameter, $a$, can be calculated from its asymptotic value, $\tilde{a}$, as

$$
a=\tilde{a} \cdot[1+f(U) \delta W / U] .
$$

BNL-79985-2007-IR

Page 8

M.T. Pigni et al. 
The Eq. 2.16 accounts for energy dependence of the level density parameter that results from the disappearance of the shell-correction $\delta W$ with increasing excitation energy as described by the $f$ function.

\subsubsection{Exciton pre-equilibrium model}

In the exciton model, the composite nucleus follows a series of more and more complicated configurations where the excited states are characterized by the number of excited particles and holes (excitons) at each stage of the pre-equilibrium cascade. Restriction to the two-body interactions leads to selection rules concerning the possible variation of the number of excitons in the course of the cascade. The initial configuration is determined by the nature of the projectile. Assuming that the pre-equilibrium systems develops only in the direction of increasing exciton number, the "never-come-back" approximation, one gets convenient closed-form expression

$$
\frac{\mathrm{d} \sigma_{a, b}}{\mathrm{~d} \epsilon}=\sigma_{a} \sum_{\Delta n=2} \mathcal{D}_{n} \frac{W_{b}(n, \epsilon)}{W_{t o t}(n)+\lambda^{+}(n)},
$$

where $\mathcal{D}_{n}$ represents the depletion factor, $W_{b}(n, \epsilon)$ is the emission rate for particle $b$ with the energy $\epsilon$ and $W_{t o t}(n)$ is the total emission rate. The internal transition rate in somewhat simplified form reads

$$
\lambda^{+}(n)=\frac{2 \pi}{\hbar}|M|^{2} g^{3} E^{2} /(n+1) .
$$

Here, $g$ is the single-particle level density, and $|M|^{2}$ is the average matrix element for the residual two-body interaction that is often parametrized through the nucleon mean free path as adopted also in the present work.

\subsubsection{Model parameters}

For the optical model we used the recent parametrization based on the extensive analysis performed by Koning and Delaroche [9] on spherical (or nearly spherical) nuclei. The energy and mass dependencies of potential parameters that were employed by the authors are more flexible than those used in previous analyses of similar type. This feature allows for a reasonable description of total and elastic cross sections as well elastic angular distributions for spherical nuclei across the periodic table. The energy range extends well above $20 \mathrm{MeV}$ adopted as the upper limit for the present exercise. In our calculations, we have used results of Ref. [9] applying relative (multiplicative factor) perturbations to the final values of the major parameters 
(potential depths, radii and diffusenesses) so that the original functional dependencies have been preserved. The varied optical model parameters are listed in Tab. 2.1, along with their respective uncertainties (3\% or 5\%), as determined by Koning from the Monte-Carlo analysis. We note, that due to the simplistic nature of the low-fidelity project, we have used spherical optical model for all considered nuclei, including the deformed ones.

Table 2.1: Parameter uncertainties used in this work for the optical model: $r$ radius, $a$ - diffuseness, $V$ - real depth, $W$ - imaginary depth. The subscripts, $v, s$ and $w$, denote real volume, real surface and imaginary surface. The superscripts, $t g \equiv \mathrm{n}+{ }_{Z}^{A}$ and $n p \equiv \mathrm{p}+{ }_{Z-1}^{A+1}$, refer to nucleon-nucleus interaction.

\begin{tabular}{|c|ccccc|}
\hline & $\Delta r_{s}^{t g}$ & $\Delta r_{v}^{t g}$ & $\Delta r_{w}^{t g}$ & $\Delta V_{v}^{t g}$ & $\Delta W_{s}^{t g}$ \\
$\%$ & \pm 3.0 & \pm 3.0 & \pm 3.0 & \pm 3.0 & \pm 5.0 \\
\hline & $\Delta W_{v}^{t g}$ & $\Delta a_{s}^{t g}$ & $\Delta a_{v}^{t g}$ & $\Delta V_{v}^{n p}$ & $\Delta W_{s}^{n p}$ \\
$\%$ & \pm 5.0 & \pm 3.0 & \pm 3.0 & \pm 3.0 & \pm 3.0 \\
\hline
\end{tabular}

Table 2.2: Parameter uncertainties used in this work for the Hauser-Feshbach and exciton models: $\tilde{a}$ - total level density, $\tilde{g}$ - single-particle level density, $f_{\gamma}$ gamma-ray strength functions, mfp - mean-free path. The superscripts refer to $c n \equiv$ compound, $t g \equiv$ target, $n 2 n \equiv(\mathrm{n}, 2 \mathrm{n})$ residue, $n p \equiv(\mathrm{n}, \mathrm{p})$ residue.

\begin{tabular}{|c|cccc|}
\hline & $\Delta \tilde{a}^{c n}$ & $\Delta \tilde{a}^{t g}$ & $\Delta \tilde{a}^{n 2 n}$ & $\Delta \tilde{a}^{n p}$ \\
$\%$ & \pm 10 & \pm 10 & \pm 10 & \pm 10 \\
\hline & $\Delta \tilde{g}^{n p}$ & $\Delta \tilde{g}^{t g}$ & $\Delta f_{\gamma}$ & $\Delta \operatorname{mfp}$ \\
$\%$ & \pm 10 & \pm 10 & \pm 20 & \pm 20 \\
\hline
\end{tabular}

The list of 8 parameters relevant for the Hauser-Feshbach and the exciton model is shown in Table 2.2. This includes four nuclear level density parameters for Hauser-Feshbach and two single-particle level densities for the exciton model, each estimated to be known within 10\%. The two remaining parameters, the $\gamma$-ray strength function and the mean-free path for the preequilibrium emission are estimated to be known within $20 \%$.

The Hauser-Feshbach statistical model is driven by nuclear level densities and the estimated global uncertainties for the related parameters are based on 
a considerable experience accumulated while using EMPIRE code in neutron cross section evaluations for the ENDF/B-VII.0 library [2]. This experience was also used to determine uncertainties for other model parameters. These estimates were finally validated by a random comparison of the calculated cross section uncertainties against the spread of experimental data. The correlations among model parameters were disregarded. This simplification is justified within the "low-fidelity" scope of the project, although it ignores some well known physical constraints (e.g., the anti-correlation between the radius $\left(r_{v}\right)$ and the real depth $\left(V_{v}\right)$ of the optical potential). Future works will have to quantify these correlations and include them in the analysis.

\subsection{Cross section sensitivities}

We performed cross section sensitivity calculations by considering uncertainties for 18 model parameters that contribute most significantly to the major reaction cross sections. The uncertainties given in Tabs. 2.1 and 2.2, define the diagonal matrix $\mathbf{P}$.

We quantify the effect of the perturbation of the model parameter $p_{j}$ on the cross section via the relative quantity

$$
\mathcal{S}\left(E_{i}, p_{j}\right)=\frac{\sigma^{+}\left(E_{i}, p_{j}\right)-\sigma^{-}\left(E_{i}, p_{j}\right)}{\sigma\left(E_{i}, \mathbf{p}\right)},
$$

where $\sigma\left(E_{i}, \mathbf{p}\right)$ is the cross section calculated for the best (or default) set of parameters $\mathbf{p}=\left(p_{1}, \ldots, p_{j}, \ldots, p_{q}\right)$, while

$$
\sigma^{ \pm}\left(E_{i}, p_{j}\right)=\sigma\left(E_{i} ; p_{1}, \ldots, p_{j} \pm \delta p_{j}, \ldots, p_{q}\right)
$$

are the cross sections calculated with the value of the parameter $p_{j}$ perturbed by its expected uncertainty $\delta p_{j}$. Then, the sensitivity matrix element $s_{i, j}$ is obtained as

$$
s_{i, j}=\mathcal{S}\left(E_{i}, p_{j}\right) \frac{\sigma\left(E_{i}, \mathbf{p}\right)}{2 \delta p_{j}}
$$

that can be viewed as the measure of the cross section response to the physically sensible variation of the model parameter $p_{j}$.

As an example, we discuss several neutron reactions on ${ }^{89} \mathrm{Y}$. Fig. 2.2 shows the response of the (n,tot), (n,el), (n,abs), (n, n'), (n, $\gamma),(n, 2 n)$, and $(n, p)$ cross sections to the variation of the real depth $\left(V_{v}^{t g}\right)$ of the optical potential 
for the target. One notes remarkably different levels of sensitivity for various reactions and strong energy dependencies. The sensitivities plotted in Fig. 2.2 are cumulative and combine effects resulting from the incident (absorption) and outgoing (inelastic scattering) channels since both use the same optical potential. The individual contributions of the two channels are shown in

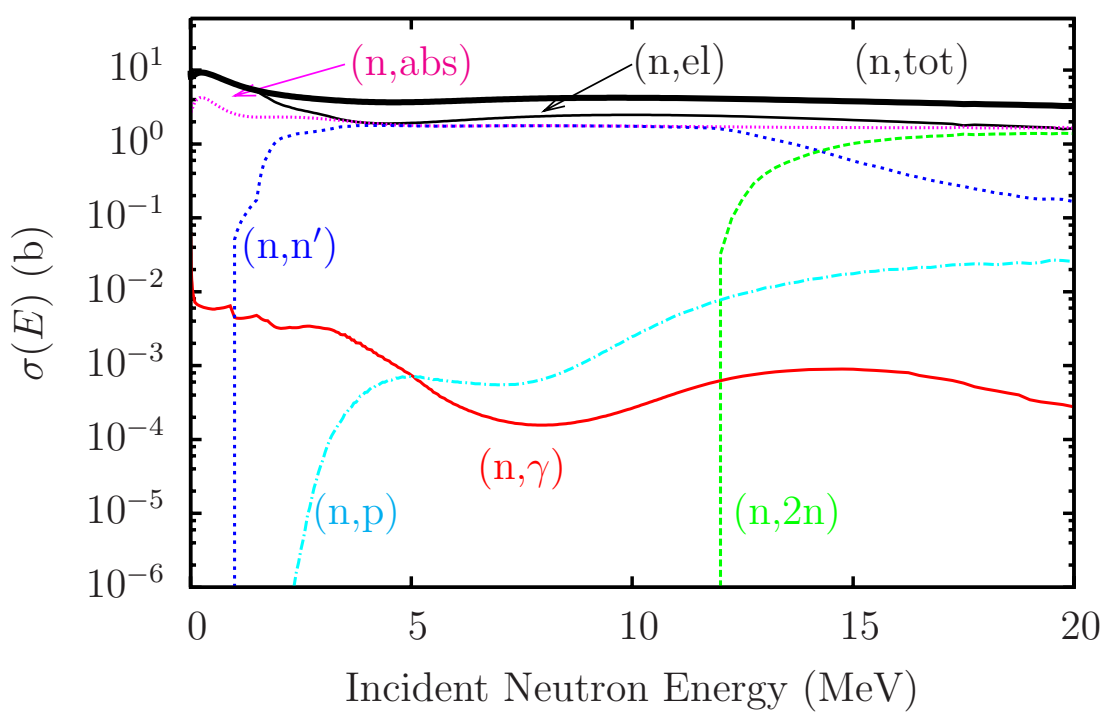

Figure 2.1: Calculated ${ }^{89} \mathrm{Y}$ neutron cross sections for different reaction channels.

Figs. 2.3 and 2.4. The salient effects that can be ascribed to the perturbation of $V_{v}^{t g}$ and summarized below:

- The $(\mathrm{n}, \gamma)$ reaction channel exhibits high sensitivity. This is partially due to the very small cross sections (see Fig. 2.1) for neutron capture on ${ }^{89} \mathrm{Y}$, which is a semi-magic nucleus (50 neutrons). Fig. 2.4 shows that the behavior of $\mathcal{S}(E, p)$ for $(\mathrm{n}, \gamma)$ reflects the competition of the inelastic scattering to the first excited states. These considerations can be extended to the $(n, p)$ reaction, which essentially presents a very similar behaviour.

- More detailed analysis shows that the dramatic sensitivity of the absorption at energies below $2 \mathrm{MeV}$ is due to the p-wave strength function which rapidly changes with the strength of the real central potential.

- The outgoing (inelastic scattering) channel is responsible for the high sensitivities at the $\left(n, n^{\prime}\right),(n, p)$, and $(n, 2 n)$ thresholds. The incoming (absorption) channel seems only to affect the sensitivity for the scattering to the first excited level in ${ }^{89} \mathrm{Y}$. 
- It is remarkable that Fig. 2.3 reveals presence of nodes (around 4 and 11 $\mathrm{MeV}$ ) at which sensitivities to the $V_{v}^{t g}$ for all reaction channels converge.

- All sensitivities change sign several times between 0 and $20 \mathrm{MeV}$. The immediate consequence of this behavior is that at these zero-crossing points the real potential depth uncertainty (even if arbitrary large) will not contribute to the cross section uncertainty.

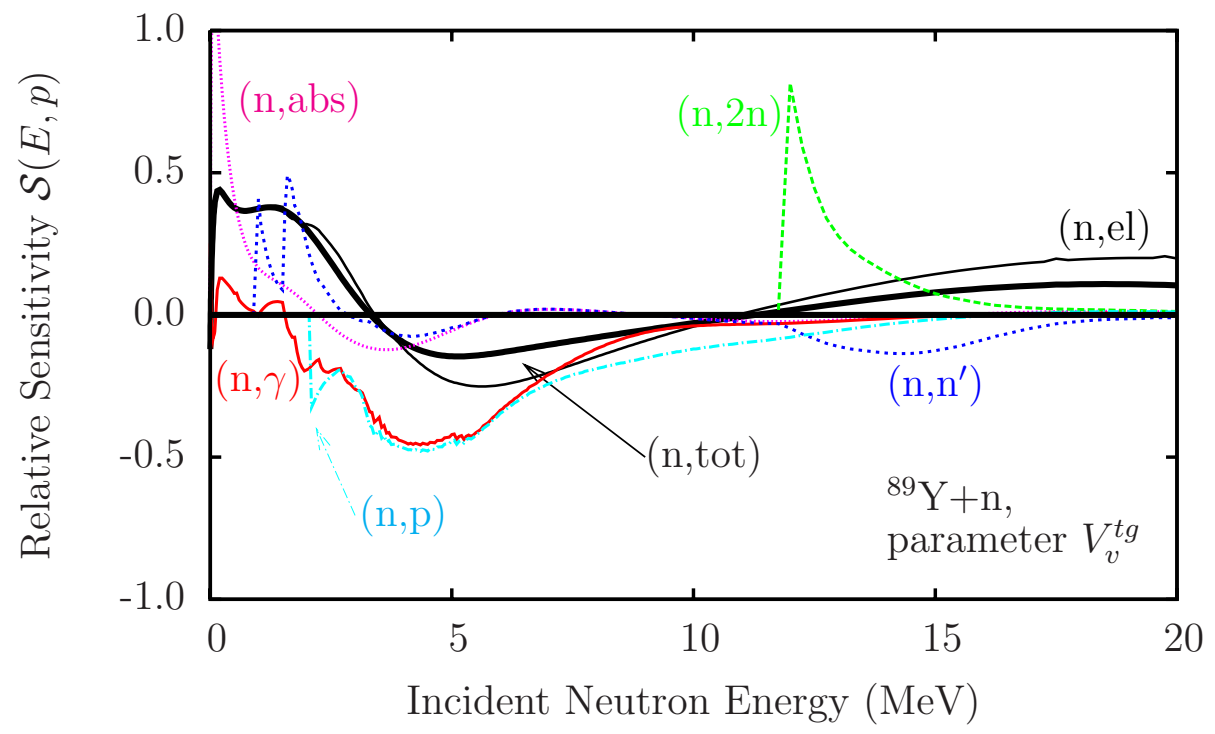

Figure 2.2: Relative sensitivity of the ${ }^{89} \mathrm{Y}+\mathrm{n}$ cross sections to the $\pm 5 \%$ perturbation of real depth $\left(V_{v}^{t g}\right)$ of the o.m.p. for neutrons. Plotted sensitivities show cumulative effects resulting from the changes in the incident (absorption) and outgoing (inelastic scattering) channels.

Fig. 2.5 shows the response of the neutron radiative capture on ${ }^{89} \mathrm{Y}$ to the variation of the most important model parameters. Two fundamental nuclear reaction mechanisms are clearly evident. In the energy region below about $10 \mathrm{MeV}$, neutron capture is well described by the formation and decay of the compound nucleus. As expected, the nuclear level density parameters $\tilde{a}^{c n}$ and $\tilde{a}^{t g}$ play an important role along with the depths of the real volume $V_{v}^{t g}$ and imaginary surface $W_{s}^{t g}$ components of the optical model potential. At higher energies the pre-equilibrium emission mechanism becomes dominant and the mean-free-path parameter ( $\mathrm{mfp}$ ) plays a major role. The effect of the radiative strength function is practically constant since $\gamma$-strength function enters both mechanisms as a multiplicative factor. 


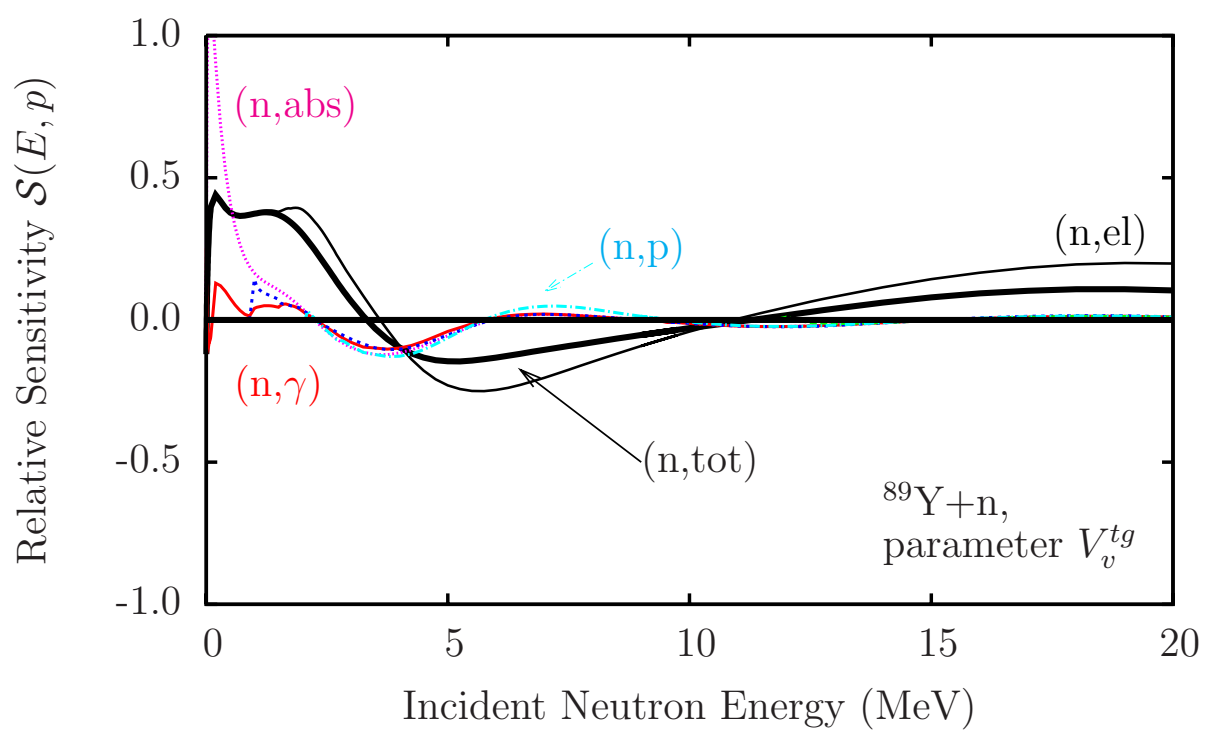

Figure 2.3: Relative sensitivity of the ${ }^{89} \mathrm{Y}+\mathrm{n}$ cross sections to the $\pm 5 \%$ perturbation of the real depth $\left(V_{v}^{t g}\right)$ o.m.p. for neutrons resulting from the changes in the incident (absorption) channel.

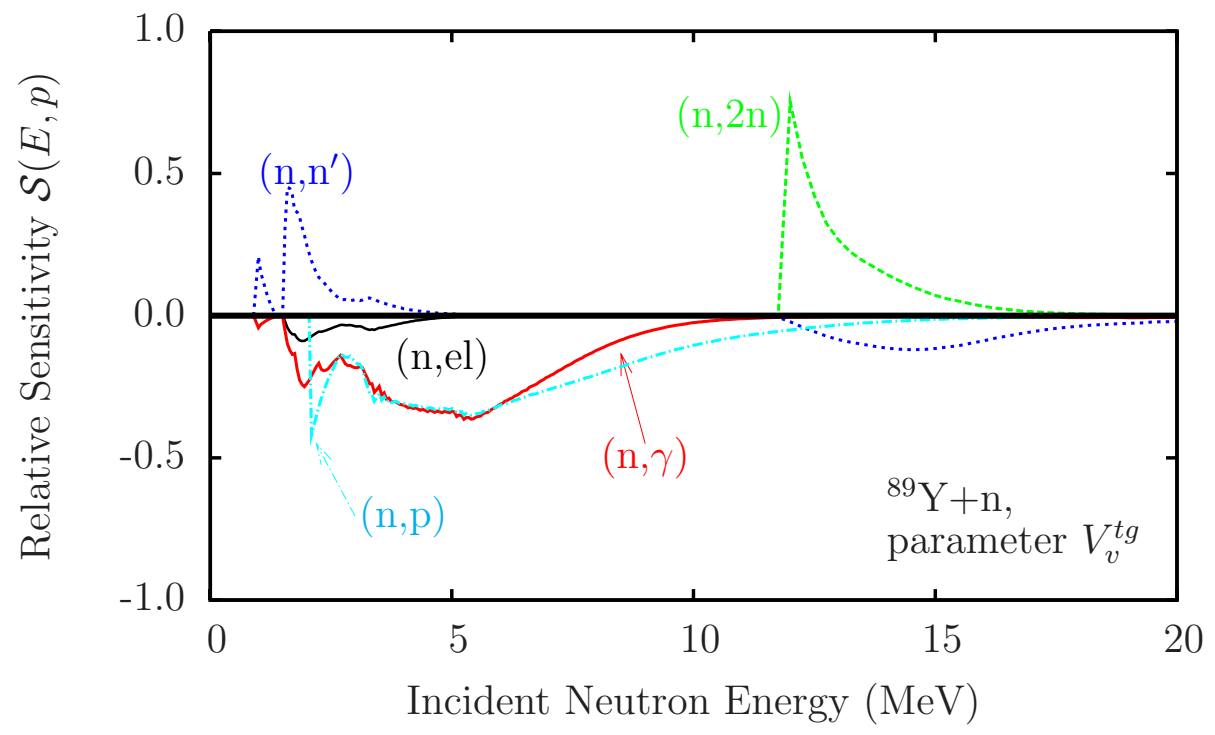

Figure 2.4: Relative sensitivity of the ${ }^{89} \mathrm{Y}+\mathrm{n}$ cross sections to the $\pm 5 \%$ perturbation of the real depth $\left(V_{v}^{t g}\right)$ o.m.p. for neutrons resulting from the changes in the outgoing (inelastic scattering) channel. 


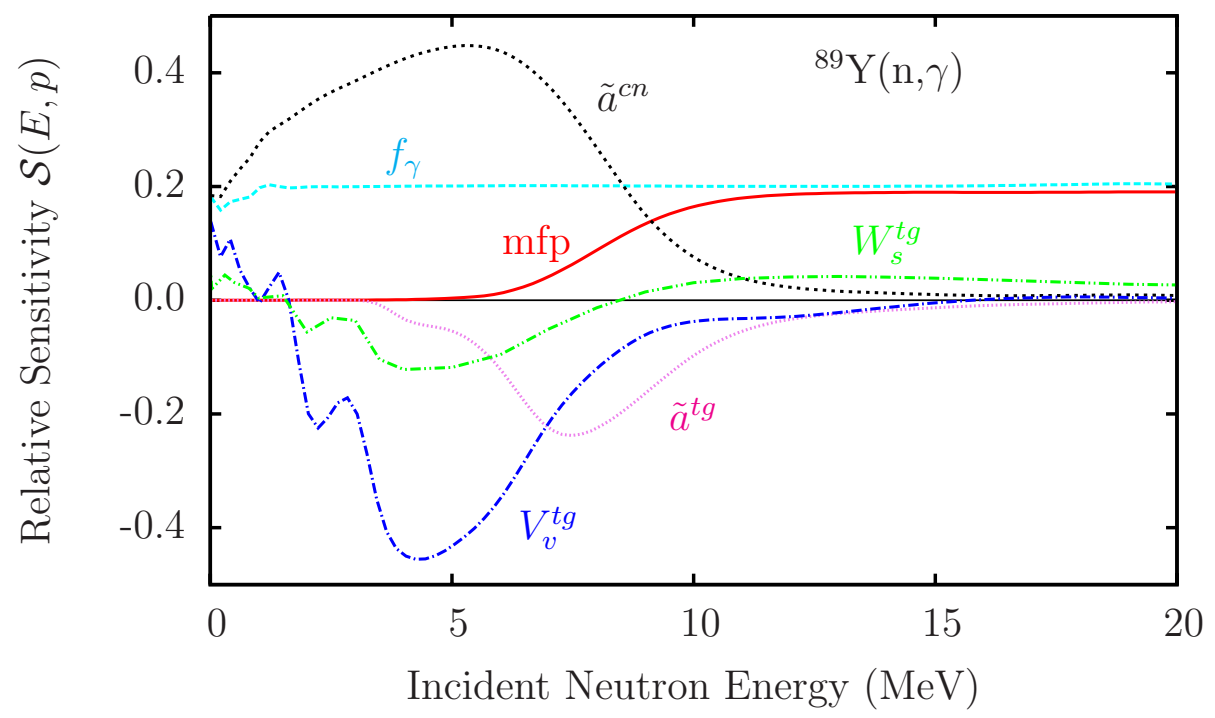

Figure 2.5: Relative sensitivity of the ${ }^{89} \mathrm{Y}(\mathrm{n}, \gamma)$ cross section to $\pm 3-10 \%$ perturbation of the level density $\left(\tilde{a}^{t g}, \tilde{a}^{c n}\right)$ and optical model parameters $\left(V_{v}^{t g}, W_{s}^{t g}\right)$, and to $\pm 20 \%$ perturbations of the $\gamma$-strength function $\left(f_{\gamma}\right)$ and the mean-free-path in the pre-equilibrium model. 


\section{Chapter 3}

\section{Calculations and Discussion}

\subsection{Calculations}

The neutron cross section covariances were calculated for 57 structural and 31 heavy isotopes at 30 incident energies between $5 \mathrm{keV}$ and $20 \mathrm{MeV}$. The five reaction channels total, elastic, inelastic, capture, and (n,2n) were considered. Altogether, 18 model parameters were varied in the calculations. The results are fully based on model calculations and while experimental data were not taken into account, they were occasionally consulted to check the quality of produced results. This is in line with the scope of the project which aims to produce low-fidelity but extensive in coverage set of covariances in a very short time.

Table 3.1: List of 307 materials evaluated by BNL. The list covers all materials in the neutron sub-library of ENDF/B-VII.0, from ${ }^{19} \mathrm{~F}$ to ${ }^{209} \mathrm{Bi}$.

\begin{tabular}{|lcc|}
\hline Materials & Nuclei & No. of isotopes \\
\hline Structural & ${ }^{19} \mathrm{~F}-{ }^{\text {nat }} \mathrm{Zn}^{*}$ & 57 \\
Fission products & ${ }^{69} \mathrm{Ga}-{ }^{170} \mathrm{Er}$ & 219 \\
Heavy & ${ }^{175} \mathrm{Lu}-{ }^{209} \mathrm{Bi}$ & 31 \\
\hline
\end{tabular}

Apart from actinides and light nuclei, we produced covariances for all materials included in the new ENDF/B-VII.0 library. This large set of 307

*The ENDF/B-VII.0 library contains neutron cross sections for elemental Zn. Considering that isotopic evaluations must be done in future, we produced covariances for a full set of $\mathrm{Zn}$ isotopes, ${ }^{64,66,67,68,70} \mathrm{Zn}$. 
materials can be divided into three regions: structural materials, fission products and heavy nuclei as summarized in Tab. 3.1. Such a distinction should guide selection of nuclear reaction modeling and parametrization. Although the scope of this project did not allow such a level of complexity, it should be applied in future refinements of our results.

\subsection{Discussion}

We will first discuss a specific example, ${ }^{56} \mathrm{Fe}$, where detailed evaluations are available in several major evaluated data libraries. Then, we will proceed with a broader, yet very useful, look on a massive amount of data that we have produced.

\subsubsection{Example - ${ }^{56} \mathrm{Fe}$}

We focus on the relative cross section uncertainties for ${ }^{56} \mathrm{Fe}+\mathrm{n}$ and start the discussion by showing our low-fidelity results. Then, we proceed with comparing these results with evaluations available in the three major evaluated data libraries, ENDF/B-VI.8, JEFF-3.1 and JENDL-3.3. We note that CSEWG decided against taking over the majority of the old covariance evaluations from the ENDF/B-VI.8 library including ${ }^{56} \mathrm{Fe}$.

Fig. 3.1 shows the relative uncertainties of the total cross section on ${ }^{56} \mathrm{Fe}$. We note that uncertainties tend to increase at low energies. In particular, we point to the presence of distinct minima in the uncertainties, which are the most striking feature in Fig. 3.1. The latter structure is related to the oscillations in the cross sections that, in turn, result from the interference of the incident neutron wave which traverses the nucleus with the wave which has been scattered. This is a quantum-mechanical feature, which is naturally incorporated in the optical model. The widths and the positions of the cross section humps are directly related to the depth of the real potential well and nuclear radius. We are currently investigating the physics background of the predicted uncertainty minima in more details and our final conclusions will be reported in a separate paper.

Fig. 3.1 compares the calculated cross sections and uncertainties with the high resolution experimental data by Harvey [10]. We show only this set to avoid cluttering the plot with a huge number of measurements available in the EXFOR library. The optical model can only predict a smooth, averaged

behaviour thus the comparison is meaningful only in the region in which there are no evident resonance-like structures. In the case of ${ }^{56} \mathrm{Fe}$ such conditions 


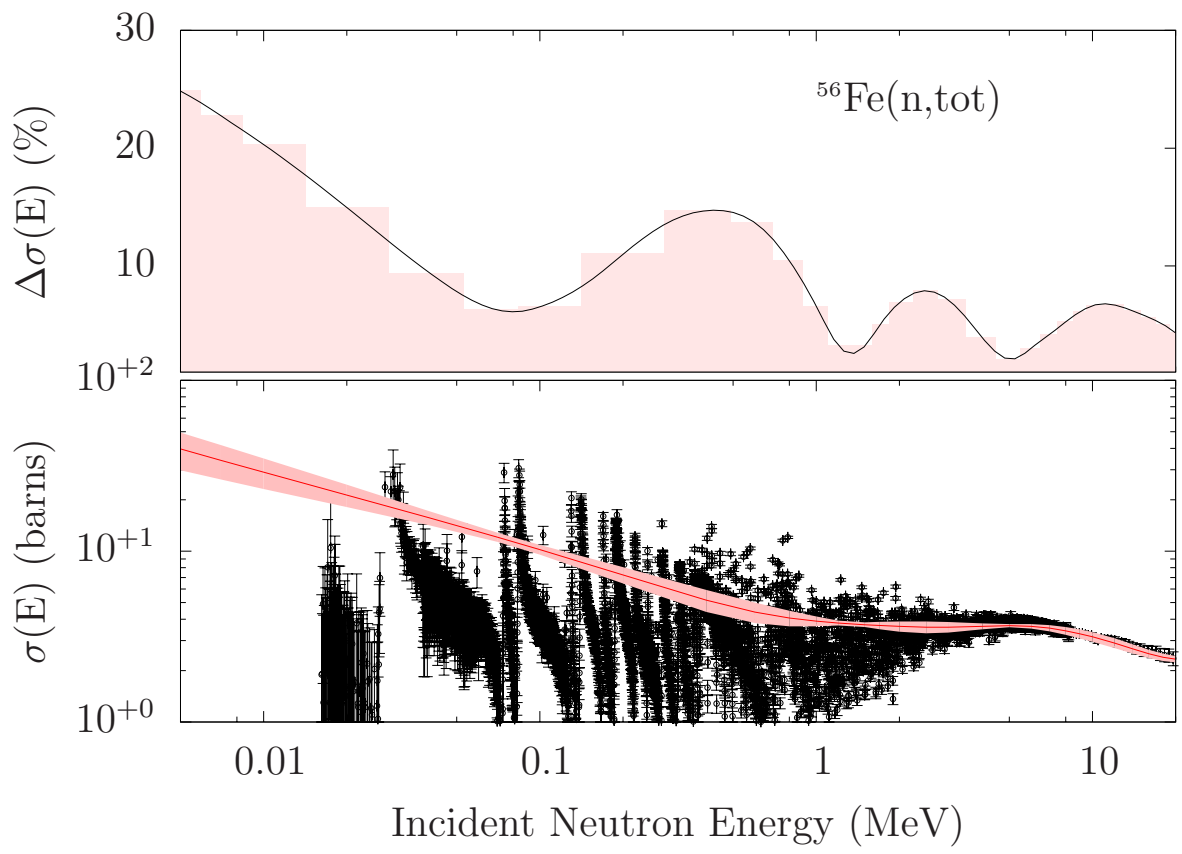

Figure 3.1: Relative uncertainties of ${ }^{56} \mathrm{Fe}(\mathrm{n}$,tot) cross sections obtained with the EMPIRE-KALMAN method. Shown are also cross sections compared with selected experimental data [10].

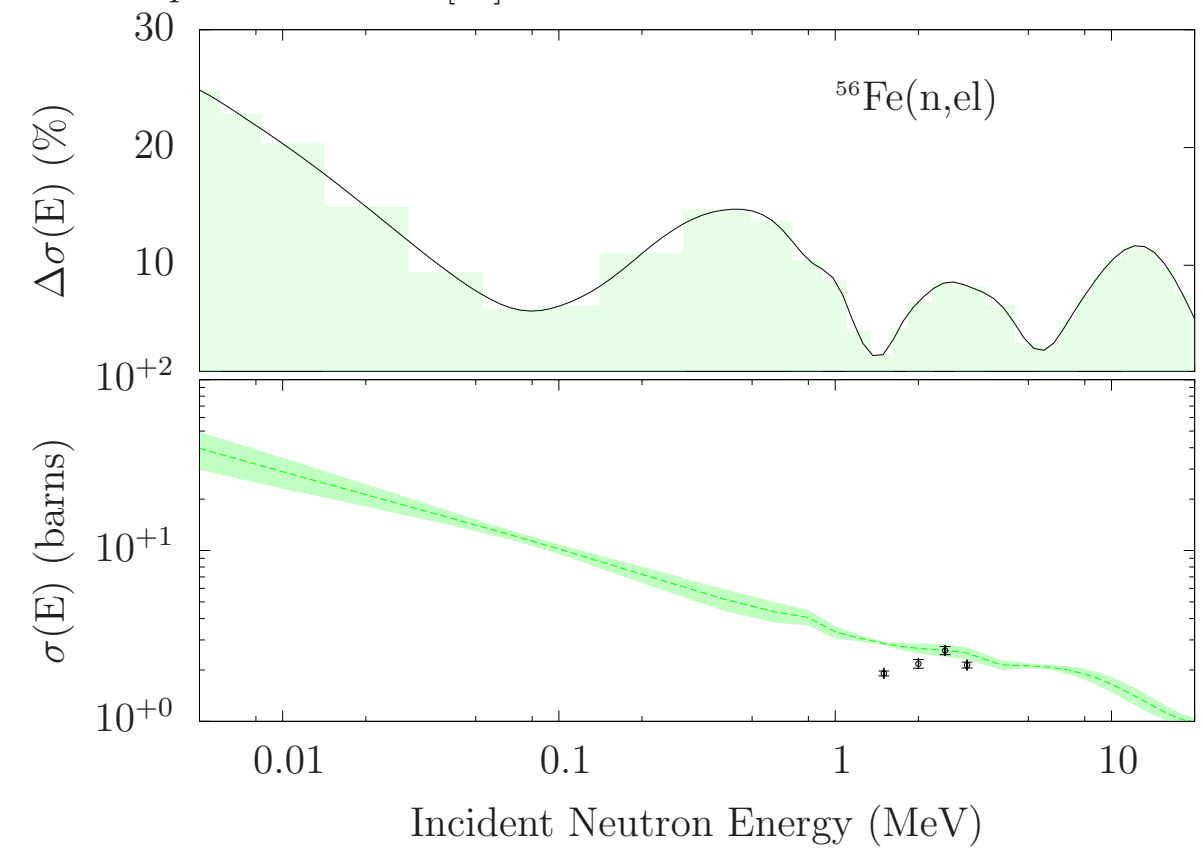

Figure 3.2: Relative uncertainties of ${ }^{56} \mathrm{Fe}(\mathrm{n}, \mathrm{el})$ cross sections obtained with the EMPIRE-KALMAN method. Shown are also cross sections compared with selected experimental data $[11,12]$. 
are reached at relatively high energies, say above $8 \mathrm{MeV}$. With this restriction in mind the comparison is quite favorable and predicted uncertainties are seem to embrace the observed scatter of experimental data. The calculated uncertainties are probably on the conservative side (over-estimated), which is in line with the "low-fidelity" scope of the project.

Relative uncertainties of neutron elastic scattering cross sections along with selected experimental data [11, 12] above $1 \mathrm{MeV}$ are shown in Fig. 3.2. The uncertainties have the same oscillating structure as for the total cross section, which could be expected since both are governed by the same optical model.

In Fig. 3.3 shows our results for the inelastic scattering. The optical model oscillations can still be noted although they appear to be much attenuated by the presence of the statistical mechanism that tends to wash out the effect of wave interference in the absorption cross section. The uncertainties turn out to increase at the threshold region and at energies above $10 \mathrm{MeV}$, while at energies between 1 and $10 \mathrm{MeV}$ they are generally lower than $10 \%$. In

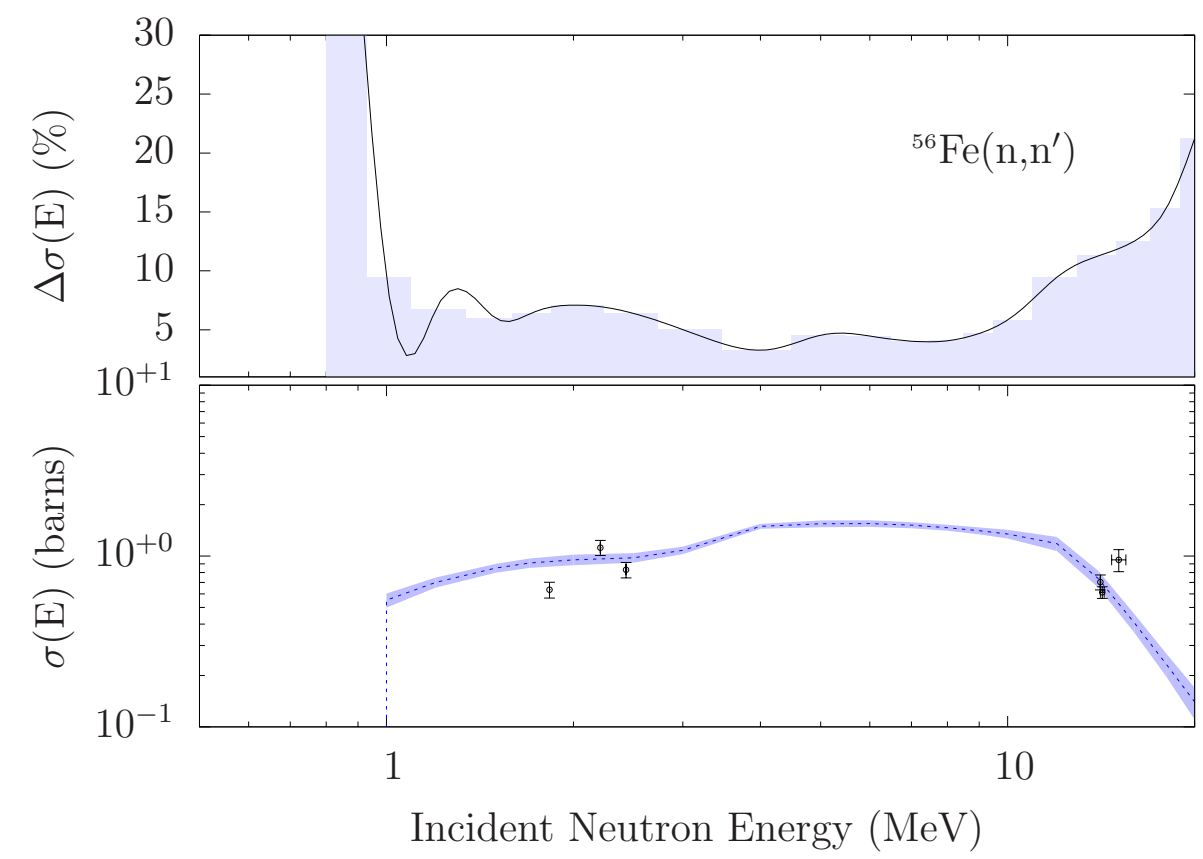

Figure 3.3: Relative uncertainties of ${ }^{56} \mathrm{Fe}\left(\mathrm{n}, \mathrm{n}^{\prime}\right)$ cross sections obtained with the EMPIRE-KALMAN method. Shown for reference are also cross sections and selected experimental data $[13,14,15,16]$.

the case of the $(\mathrm{n}, 2 \mathrm{n})$ reaction (Fig. 3.4) the uncertainties are essentially flat outside the threshold region. We note, that the experimental cross sections fall within the calculated error band. The optical model oscillations are not visible. 


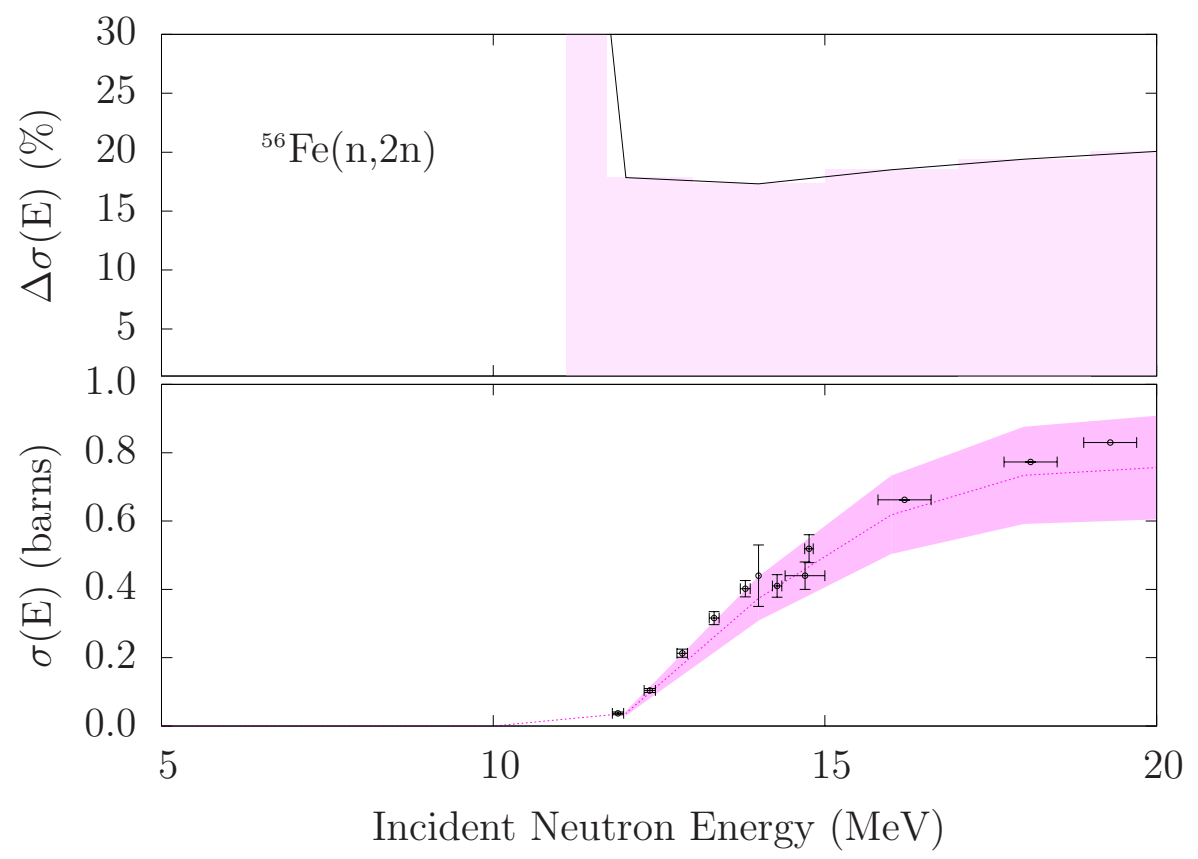

Figure 3.4: Relative uncertainties of ${ }^{56} \mathrm{Fe}(\mathrm{n}, 2 \mathrm{n})$ cross sections obtained with the EMPIRE-KALMAN method. Shown for reference are also cross sections and selected experimental data $[17,18,19]$.

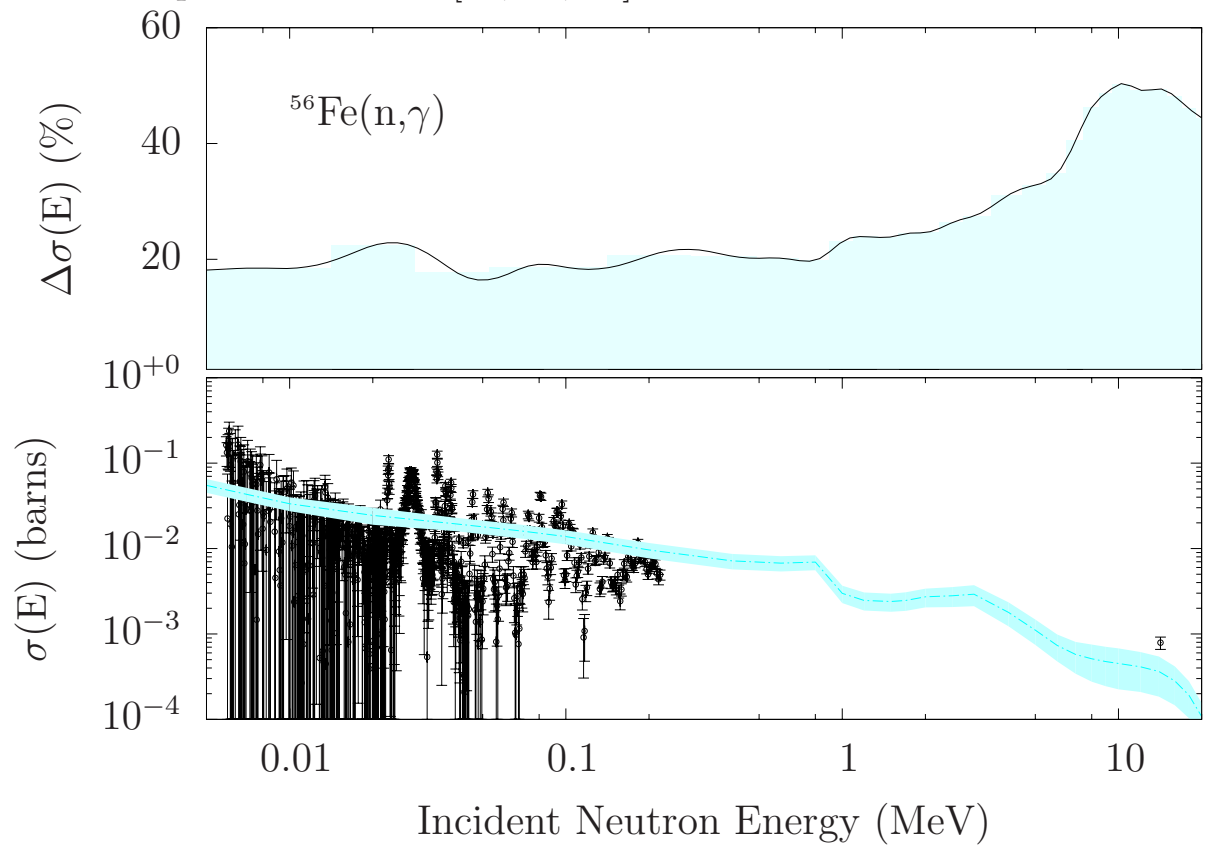

Figure 3.5: Relative uncertainties of ${ }^{56} \mathrm{Fe}(\mathrm{n}, \gamma)$ cross sections obtained with the EMPIRE-KALMAN method. Shown are also cross sections and the high resolution experimental data [20,21, 22]. 
Fig. 3.5 summarizes the results for the capture cross sections by showing uncertainties as well as calculated cross sections along with the experimental data. It is obvious that the calculated capture cross sections cannot reproduce experimental fluctuations observed in the high resolution experiments below $0.1 \mathrm{MeV}$. Therefore, the uncertainty ( $\gtrsim 20 \%)$ should be interpreted as an average over broader energy range and not point-wise. As expected, uncertainties rise above $1 \mathrm{MeV}$.

In Fig. 3.6, we show an example of the full correlation matrix for ${ }^{56} \mathrm{Fe}(\mathrm{n}, \mathrm{tot})$ together with relative cross section uncertainties. Since the correlation matrices are symmetric, we show only the part below diagonal.

Nuclear applications, including criticality safety, require neutron covariance matrices in the multi-group representation. These are just the averages of the point-wise covariance matrices, i.e., $\left\langle\Delta \sigma(E) \Delta \sigma\left(E^{\prime}\right)\right\rangle$, over union groups I, J,

$$
\left\langle\Delta \sigma_{I} \Delta \sigma_{J}\right\rangle=\frac{\int_{I} \int_{J} \phi(E) \phi\left(E^{\prime}\right)\left\langle\Delta \sigma(E) \Delta \sigma\left(E^{\prime}\right)\right\rangle \mathrm{d} E \mathrm{~d} E^{\prime}}{\int_{I} \int_{J} \phi(E) \phi\left(E^{\prime}\right) \mathrm{d} E \mathrm{~d} E^{\prime}},
$$

where $\phi(E)$ is the flux "model" assumed for the multi-group calculations. It is useful to compare our low-fidelity cross section uncertainties, e.g., for ${ }^{56} \mathrm{Fe}+\mathrm{n}$, with multi-group evaluations performed for the three major nuclear data libraries. Our results are given in the point-wise form that includes 30 energy points above $5 \mathrm{keV}$. The results from the evaluated libraries were extracted by the Los Alamos code NJOY and processed into 44-energy group representation. We note, that only a limited number of groups in this representation overlap with our energy range of $5 \mathrm{keV}-20 \mathrm{MeV}$. We also note, that positive correlations decrease the multi-group uncertainties below the point-wise values.

Figure 3.7 illustrates our low-fidelity results by showing ${ }^{56} \mathrm{Fe}$ (n,tot) cross section uncertainties and comparing them to those in the ENDF/B-VI.8, JEFF-3.1 and JENDL-3.3 evaluations (the latter in the 44-energy group representation). As expected, the low-fidelity uncertainties are generally higher than the multi-group results from other libraries, particularly in the lower energy range. This discrepancy can easily be explained. First of all, contrary to the regular evaluations, our estimates do not make explicit use of the experimental data and rely exclusively on the model calculations using global parameters. It happens that sensitivity to the optical model parameters is 
Printed by NNDC

\begin{tabular}{|lcc} 
Dec 11, 07 11:13 & mtrx-tot.d & Page 1/1 \\
\hline
\end{tabular}




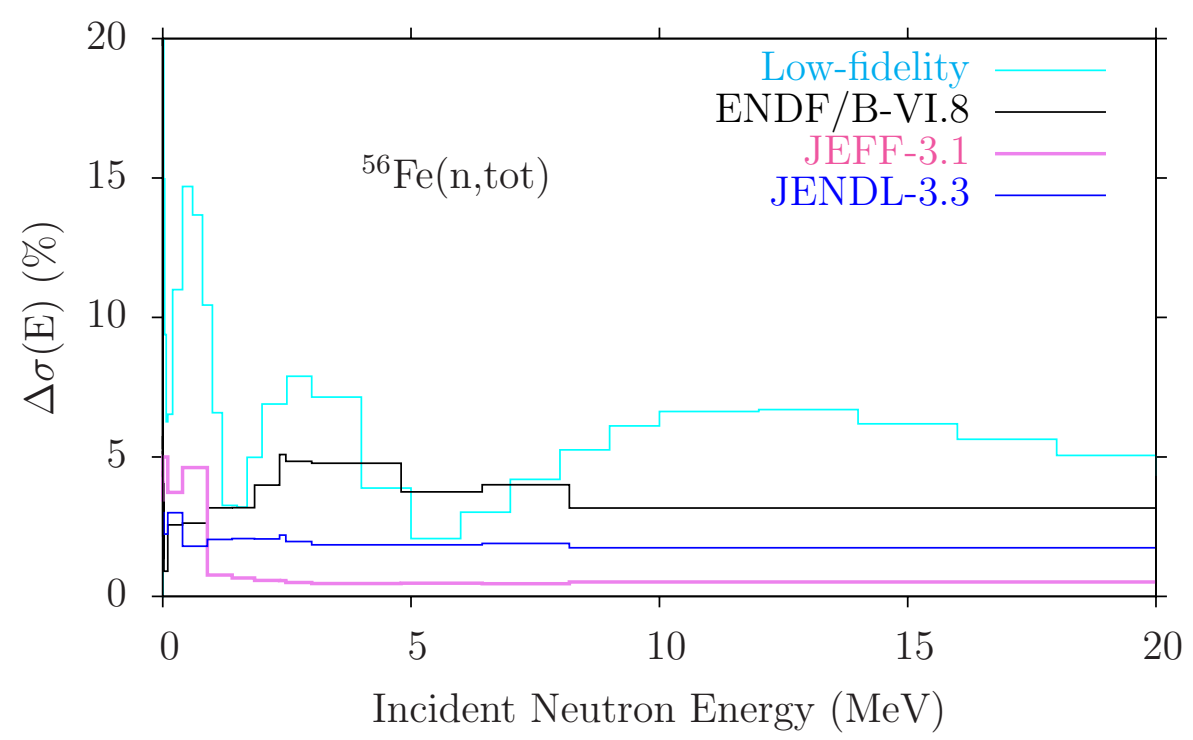

Figure 3.7: Relative uncertainties of ${ }^{56} \mathrm{Fe}(\mathrm{n}$,tot) cross sections compared to three major nuclear data libraries. Our low-fidelity results are in the point-wise form, while the other data are in the 44-energy group representation.

particularly high at low energies and this translates into high cross section uncertainties. Accidentally, these higher uncertainties happen to occur in the region where cross sections fluctuate due to the resonance structure. Therefore, the big uncertainty is not unreasonable when ascribed to the smooth optical model cross section that replaces fluctuating reality. On the other hand, true evaluations tend to reproduce experimental structure, thus are much closer to the reality than optical model predictions. This justifies much lower uncertainties reported in the national libraries. Generally, we should find lower uncertainties whenever there is a wealth of experimental data that have been used in the evaluation.

Further comparison between multi-group evaluations and our low-fidelity results is illustrated in Figure 3.9 and 3.8. Except of the low energy region, our results for elastic and inelastic scattering uncertainties are in a reasonable agreement with other evaluations. On the contrary, the uncertainties for the $(n, 2 n)$ reaction channel in Fig. 3.10 are strongly overestimated in the whole energy range. This is simply due to the fact that extensive experimental information available for the ${ }^{56} \mathrm{Fe}(\mathrm{n}, 2 \mathrm{n})$ reaction, which was driving other evaluations, was totally ignored in our estimates. We recall, that the basic concept of the present exercise was provide a complete set of approximate covariances derived from the global nuclear model calculations. Finally, in Fig. 3.11 we compare cross section uncertainties for the ${ }^{56} \mathrm{Fe}(\mathrm{n}, \gamma)$ 
reaction. The results are in acceptable agreement in view of the multi-group representation. In particularly, we note the same energy behavior as in the JENDL-3.3 library. It confirms our previous finding that the agreement between low-fidelity and other evaluations is reasonable whenever there is little experimental evidence available for analysis. 


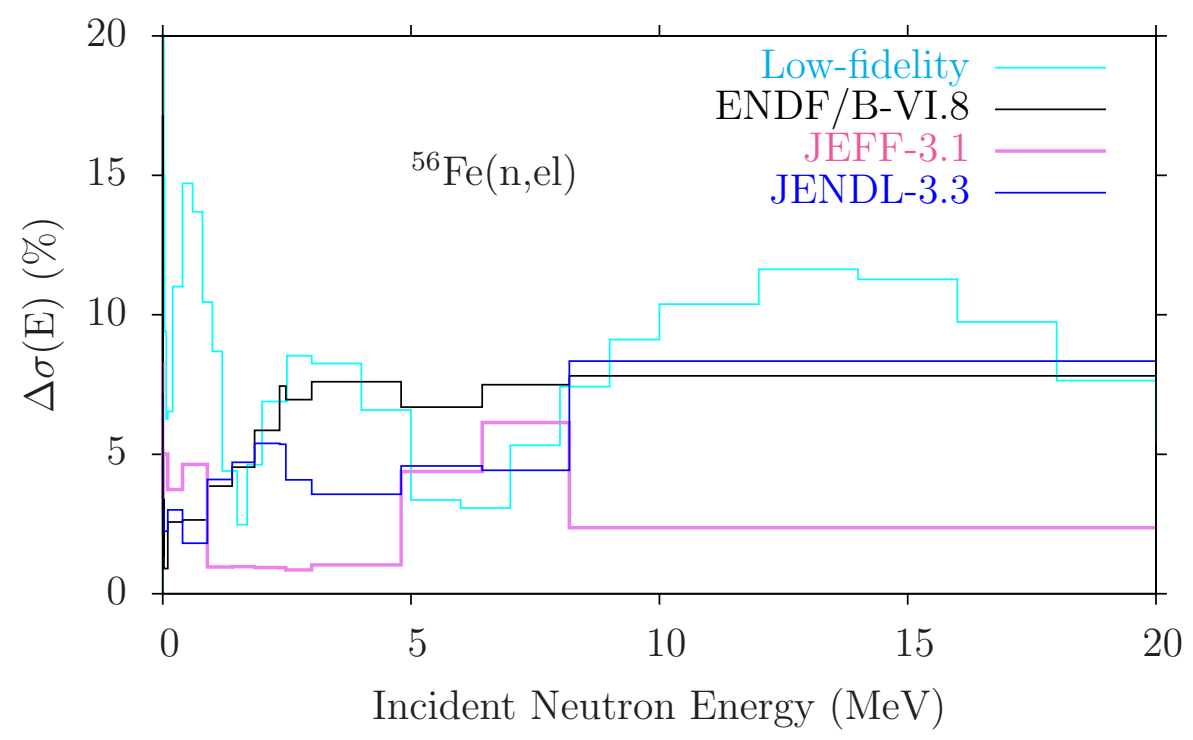

Figure 3.8: Relative uncertainties of ${ }^{56} \mathrm{Fe}(\mathrm{n}, \mathrm{el})$ cross sections compared to three major nuclear data libraries. Our low-fidelity results are in the point-wise form, while the other data are in the 44-energy group representation.

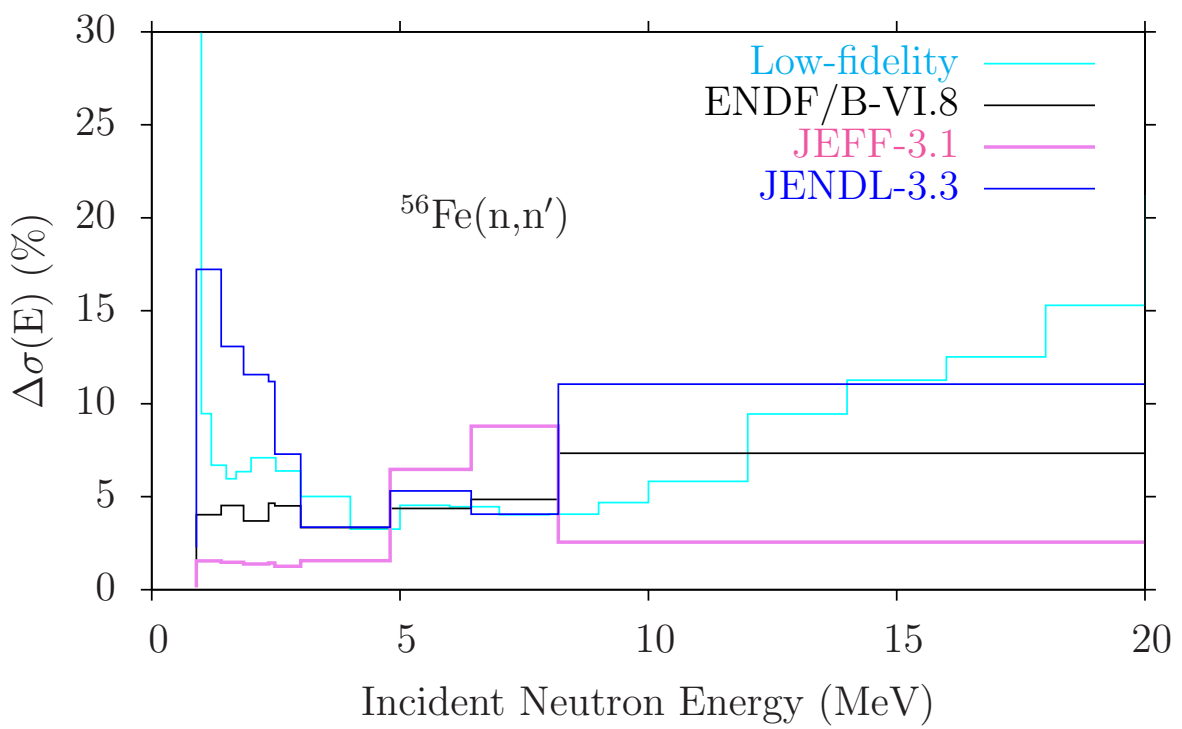

Figure 3.9: Relative uncertainties of ${ }^{56} \mathrm{Fe}\left(\mathrm{n}, \mathrm{n}^{\prime}\right)$ cross sections compared to three major nuclear data libraries. Our low-fidelity results are in the point-wise form, while the other data are in the 44-energy group representation. 


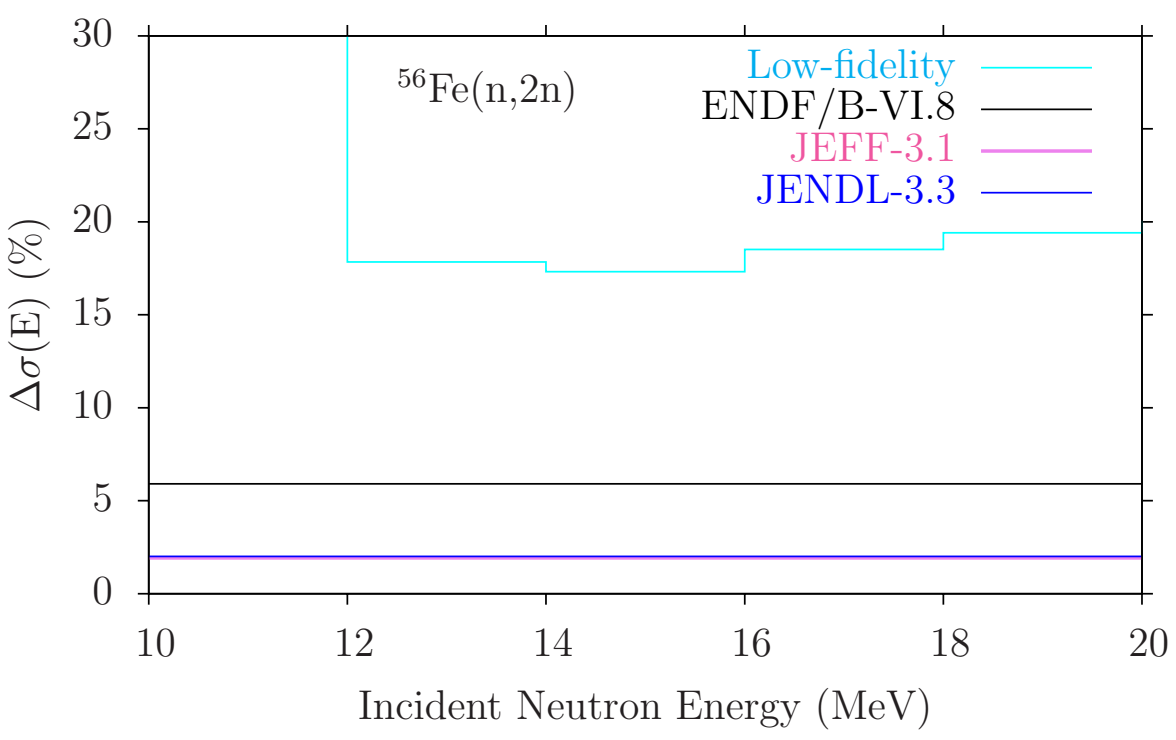

Figure 3.10: Relative uncertainties of ${ }^{56} \mathrm{Fe}(\mathrm{n}, 2 \mathrm{n})$ cross sections compared to three major nuclear data libraries. Our low-fidelity results are in the point-wise form, while the other data are in the 44-energy group representation.

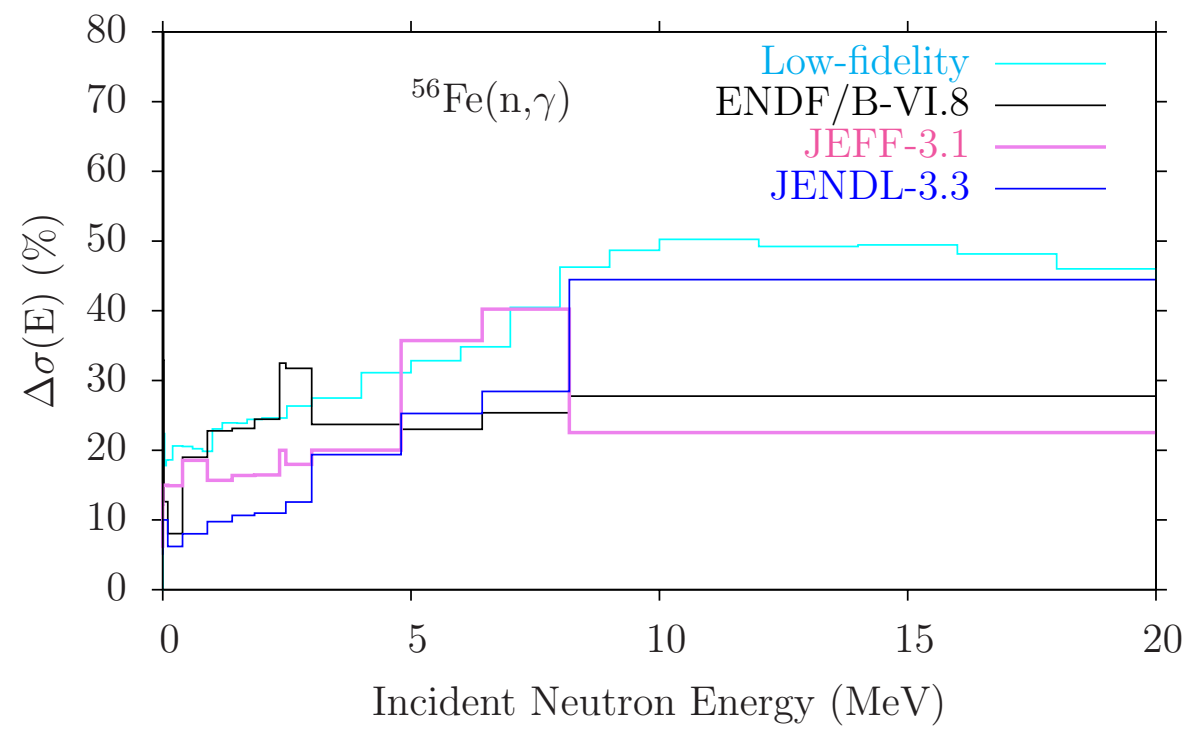

Figure 3.11: Relative uncertainties of ${ }^{56} \mathrm{Fe}(\mathrm{n}, \gamma)$ cross sections compared to three major nuclear data libraries. Our low-fidelity results are in the point-wise form, while the other data are in the 44-energy group representation. 


\subsubsection{Materials from ${ }^{19} \mathrm{~F}$ to ${ }^{209} \mathrm{Bi}$}

To present the overall picture of our results we developed contour plots that show full set of nuclei in the full range of incident energies on a single plot. These plots show relative cross section uncertainties represented by different colors, from $0 \%$ shown in black to $100 \%$ shown in yellow.Using these plots, we show in Figs. 3.12-3.16 the relative cross section uncertainties for the major reaction channels. The $\mathrm{x}$ - and $\mathrm{y}$-axes refer to the mass numbers of the complete list of 307 materials and to all incident neutron energies, respectively.

Figures 3.12 and 3.13 show total and neutron elastic scattering channels. For both exceptionally high uncertainties are found for nuclei between Xe and $\mathrm{Eu}$ at incident energies below $100 \mathrm{keV}$. A possible explanation of this effect can be traced to the structure observed in the s- and/or d-wave neutron strength functions. For these two reaction channels we also note very similar patterns characterized by regions where the uncertainties are particularly small. These intriguing structures derive from the position of the minima already discussed in the case of ${ }^{56} \mathrm{Fe}$, see Figs. 3.1 and 3.2.

In Fig. 3.14, we show a summary of neutron inelastic scattering by providing a contour plot for the complete list of materials. Inelastic scattering is the threshold reaction, hence, black color at below thresholds reflects perfect knowledge of the zero cross section. Some deviations from this pattern can be explained by metastable targets where the threshold for inelastic scattering is zero. At intermediate energies some structure is observed, while at the highest energies, as the cross sections drop, the model predictions become poor (shown as yellow areas).

Shown in Fig. 3.15 are the cross section uncertainties for the (n,2n) reactions. The relative uncertainties are basically flat but one may note an isotopic effect represented on the plot by vertical lines of different intensity.

In Fig. 3.16, we show a summary of the uncertainties for the neutron capture cross sections. While relatively good precision can be achieved at lower energies, the uncertainties increase with the incident neutron energy. Above $10 \mathrm{MeV}$ capture cross sections represent such tiny fraction of the absorption that model predicted uncertainties exceed $50 \%$. We stress, that this result is only due to the propagation of model parameter uncertainties and does not include numerical (rounding) errors. The latter are not supposed to be a major issue for the capture reactions below $20 \mathrm{MeV}$ but are known to create problems in the $(\mathrm{n}, \alpha)$ reactions close to thresholds. 


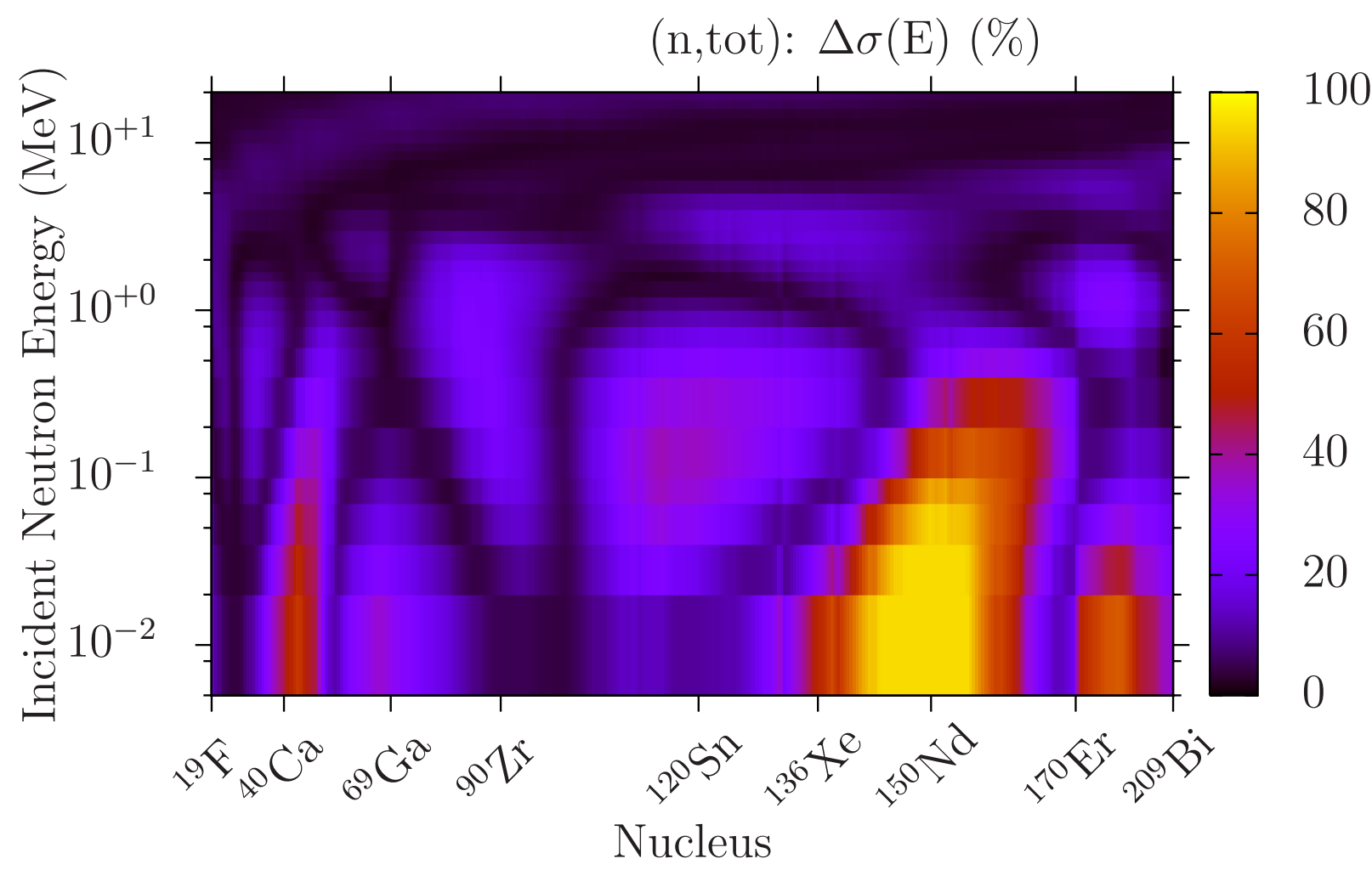

Figure 3.12: Relative uncertainties for the total cross sections on 307 materials obtained with the EMPIRE-KALMAN method in the fast neutron energy region. 


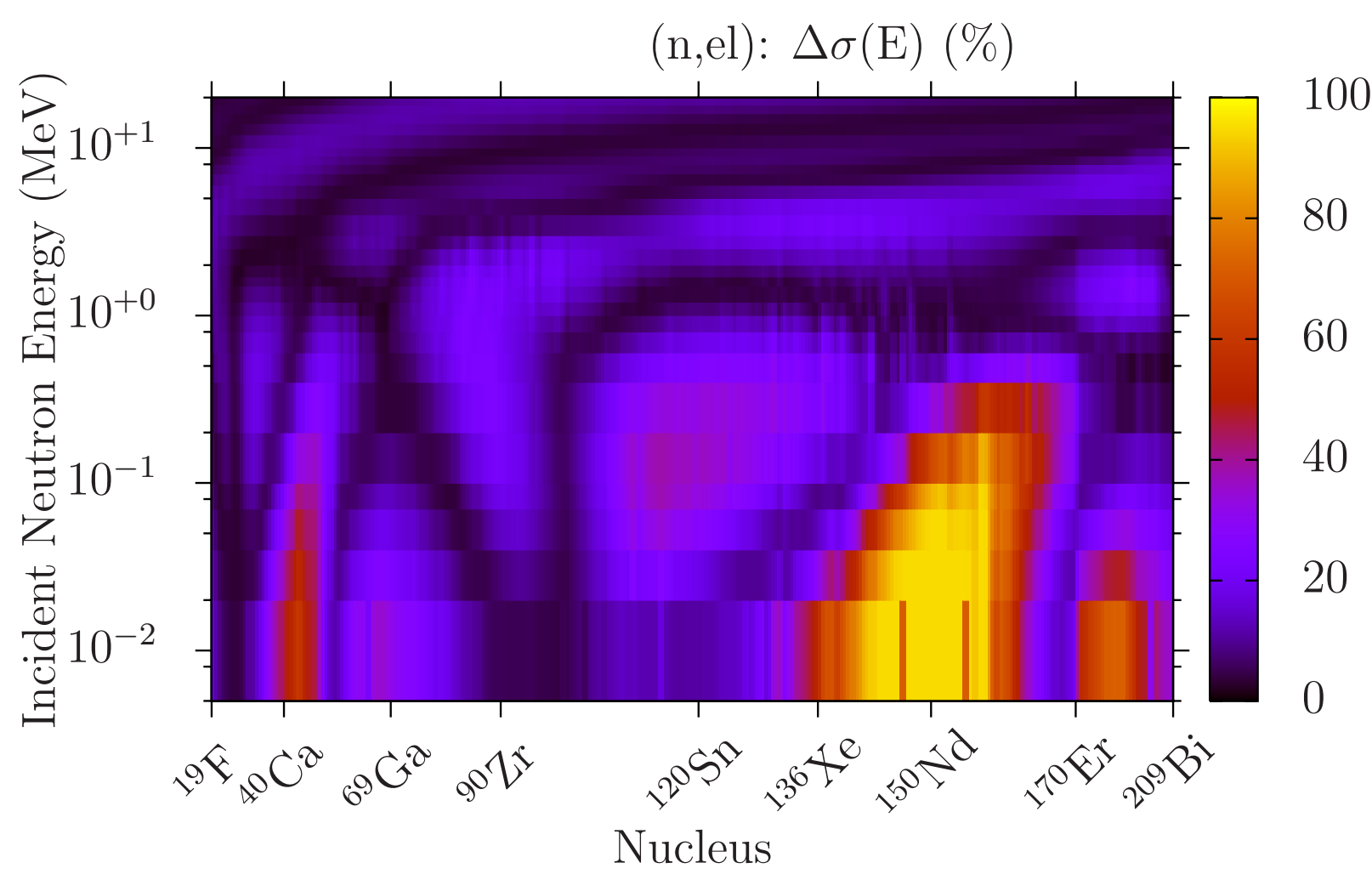

Figure 3.13: Relative uncertainties for the elastic cross sections on 307 materials obtained with the EMPIRE-KALMAN method in the fast neutron energy region. 


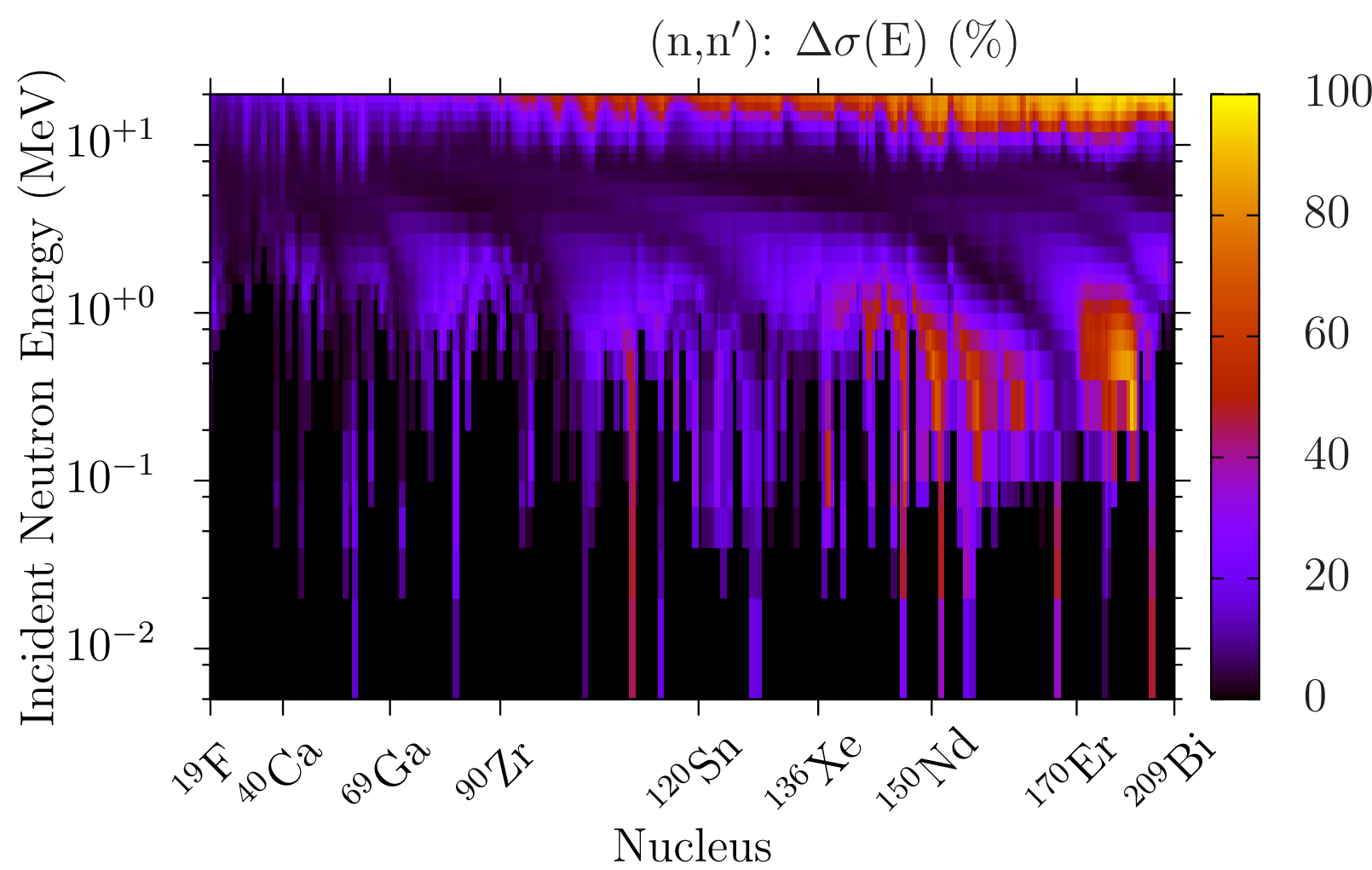

Figure 3.14: Relative uncertainties for the inelastic cross sections on 307 materials obtained with the EMPIRE-KALMAN method in the fast neutron energy region. 


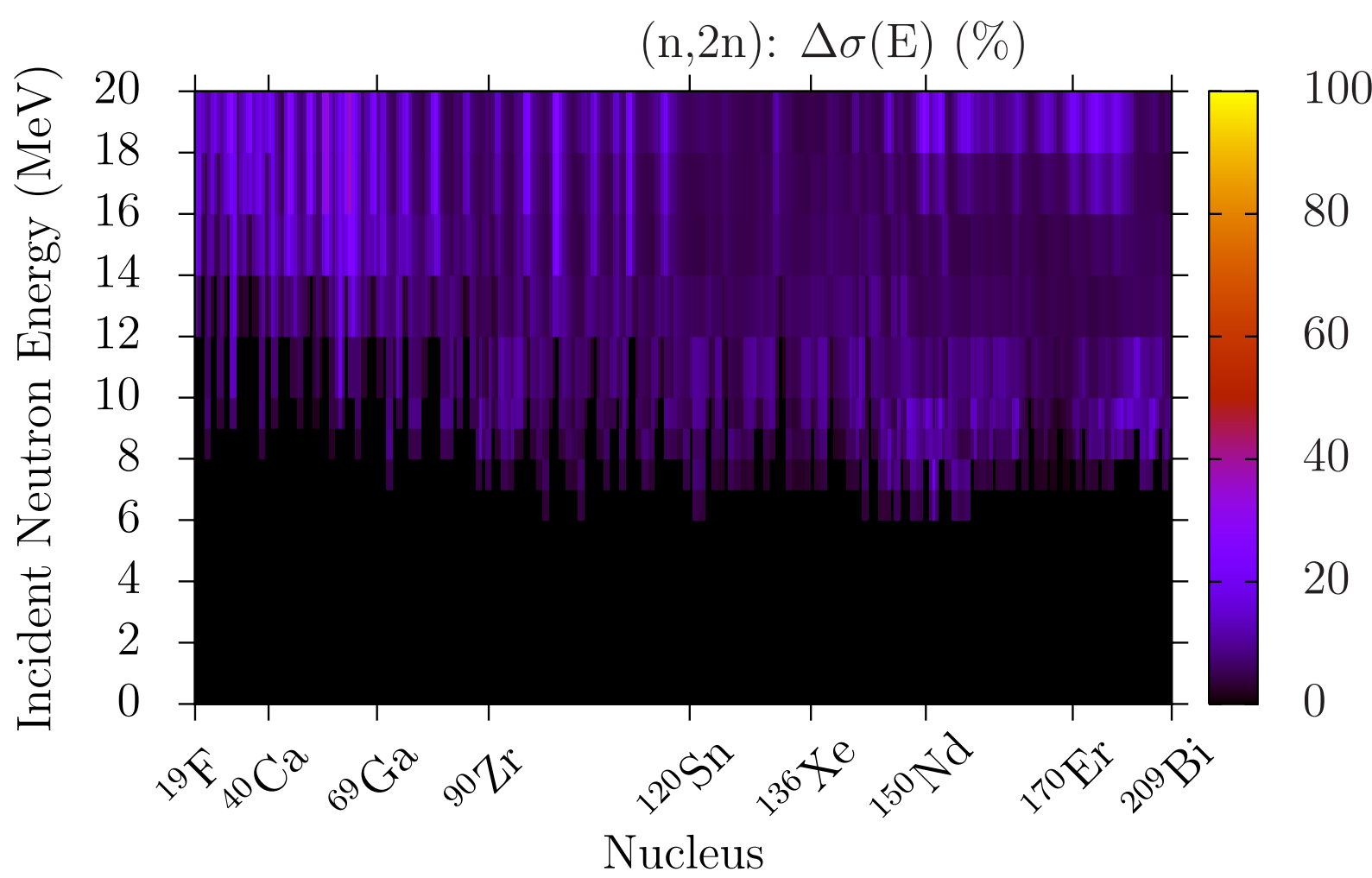

Figure 3.15: Relative uncertainties for the $(n, 2 n)$ cross sections on 307 materials obtained with the EMPIRE-KALMAN method in the fast neutron energy region. 


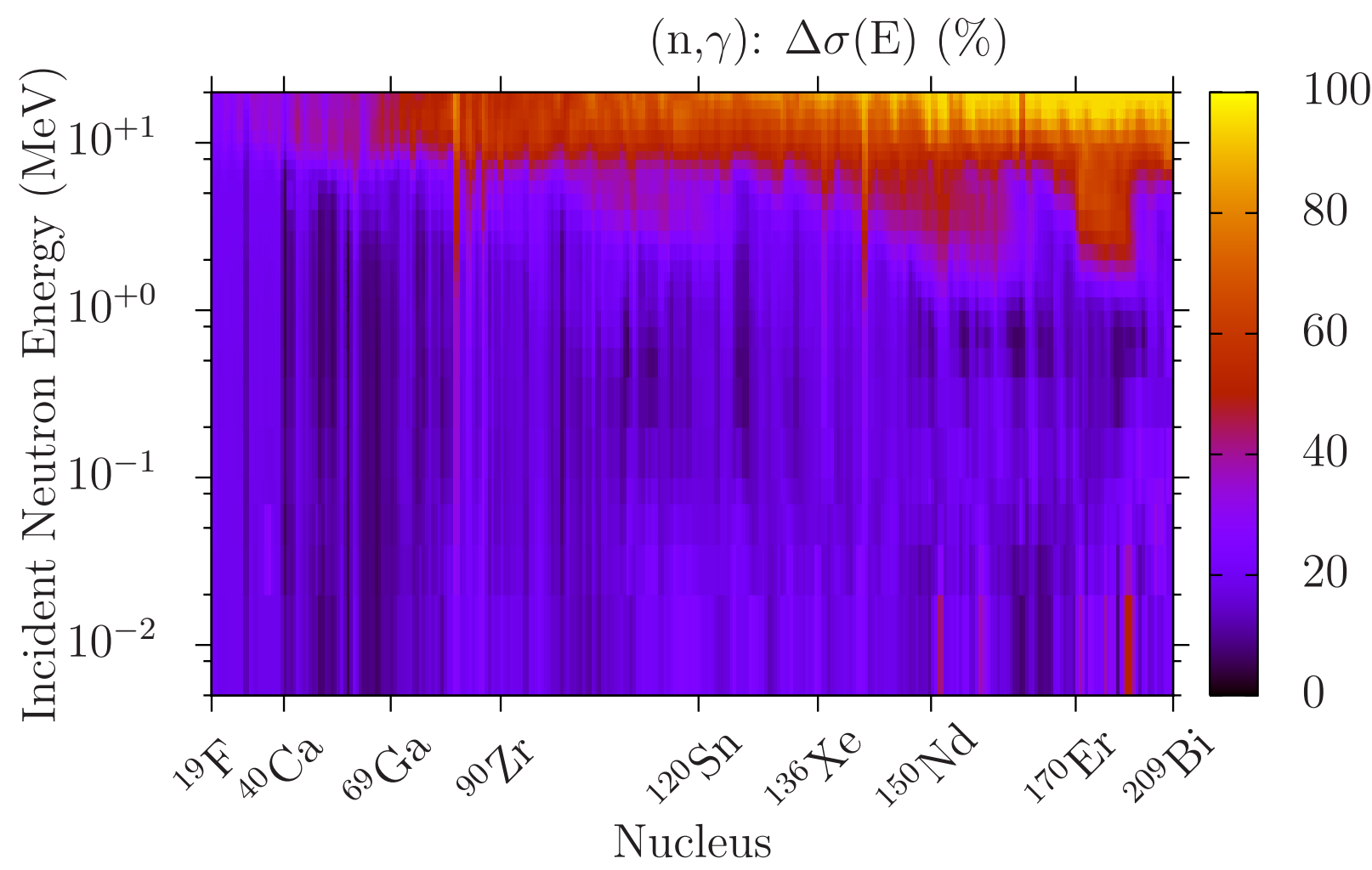

Figure 3.16: Relative uncertainties for the capture cross sections on 307 materials obtained with EMPIRE-KALMAN method in the fast neutron energy region. 


\section{Chapter 4}

\section{Results}

The neutron cross section covariance matrices were calculated for 57 structural and 31 heavy nuclei at 30 neutron incident energies between $5 \mathrm{keV}$ and $20 \mathrm{MeV}$ (see Tab. 4.1). The four reaction channels considered in the present work were elastic, inelastic, capture, and $(n, 2 n)$. Altogether, 18 model parameters were varied in the calculations. The results are completely based on model calculations; no experimental data were taken into account. The uncertainties were determined following the procedure presented in Section 2.1.

The list of 57 structural and 31 heavy nuclei is given in Tabs. 4.2 and 4.3, respectively.

Table 4.1: The list of 30 neutron incident energies used in the calculations of cross section covariances.

\begin{tabular}{|clclcl|}
\hline No. & Energy (MeV) & No. & Energy (MeV) & No. & Energy $(\mathrm{MeV})$ \\
\hline 1 & 0.005 & 11 & 0.800 & 21 & 6.000 \\
2 & 0.007 & 12 & 1.000 & 22 & 7.000 \\
3 & 0.010 & 13 & 1.200 & 23 & 8.000 \\
4 & 0.020 & 14 & 1.500 & 24 & 9.000 \\
5 & 0.040 & 15 & 1.700 & 25 & 10.00 \\
6 & 0.070 & 16 & 2.000 & 26 & 12.00 \\
7 & 0.100 & 17 & 2.500 & 27 & 14.00 \\
8 & 0.200 & 18 & 3.000 & 28 & 16.00 \\
9 & 0.400 & 19 & 4.000 & 29 & 18.00 \\
10 & 0.600 & 20 & 5.000 & 30 & 20.00 \\
\hline
\end{tabular}


Table 4.2: List of structural materials evaluated by BNL. The list corresponds to the neutron sub-library of the ENDF/B-VII.0 library [2].

\begin{tabular}{|c|c|c|c|c|c|}
\hline No. & Material & MAT & No. & Material & MAT \\
\hline 1) & ${ }^{19} \mathrm{~F}$ & 925 & 29) & ${ }^{48} \mathrm{Ca}$ & 2049 \\
\hline 2) & ${ }^{22} \mathrm{Na}$ & 1122 & 30) & ${ }^{45} \mathrm{Sc}$ & 2125 \\
\hline 3) & ${ }^{23} \mathrm{Na}$ & 1125 & $31)$ & ${ }^{46} \mathrm{Ti}$ & 2225 \\
\hline 4) & ${ }^{24} \mathrm{Mg}$ & 1225 & $32)$ & ${ }^{47} \mathrm{Ti}$ & 2228 \\
\hline 5) & ${ }^{25} \mathrm{Mg}$ & 1228 & $33)$ & ${ }^{48} \mathrm{Ti}$ & 2231 \\
\hline 6$)$ & ${ }^{26} \mathrm{Mg}$ & 1231 & 34) & ${ }^{49} \mathrm{Ti}$ & 2234 \\
\hline 7) & ${ }^{27} \mathrm{Al}$ & 1325 & $35)$ & ${ }^{50} \mathrm{Ti}$ & 2237 \\
\hline 8) & ${ }^{28} \mathrm{Si}$ & 1425 & $36)$ & ${ }^{51} \mathrm{~V}$ & 2300 \\
\hline 9) & ${ }^{29} \mathrm{Si}$ & 1428 & $37)$ & ${ }^{50} \mathrm{Cr}$ & 2425 \\
\hline 10) & ${ }^{30} \mathrm{Si}$ & 1431 & $38)$ & ${ }^{52} \mathrm{Cr}$ & 2431 \\
\hline 11) & ${ }^{31} \mathrm{P}$ & 1525 & 39) & ${ }^{53} \mathrm{Cr}$ & 2434 \\
\hline 12) & ${ }^{32} \mathrm{~S}$ & 1625 & 40) & ${ }^{54} \mathrm{Cr}$ & 2437 \\
\hline 13) & ${ }^{33} \mathrm{~S}$ & 1628 & 41) & ${ }^{55} \mathrm{Mn}$ & 2525 \\
\hline 14) & ${ }^{34} \mathrm{~S}$ & 1631 & 42) & ${ }^{54} \mathrm{Fe}$ & 2625 \\
\hline 15) & ${ }^{36} \mathrm{~S}$ & 1637 & 43) & ${ }^{56} \mathrm{Fe}$ & 2631 \\
\hline 16) & ${ }^{35} \mathrm{Cl}$ & 1725 & 44) & ${ }^{57} \mathrm{Fe}$ & 2634 \\
\hline 17) & ${ }^{37} \mathrm{Cl}$ & 1731 & $45)$ & ${ }^{58} \mathrm{Fe}$ & 2637 \\
\hline 18) & ${ }^{36} \mathrm{Ar}$ & 1825 & 46) & ${ }^{58} \mathrm{Co}$ & 2722 \\
\hline 19) & ${ }^{38} \mathrm{Ar}$ & 1831 & 47) & ${ }^{58} \mathrm{Co}$ & 2723 \\
\hline 20) & ${ }^{40} \mathrm{Ar}$ & 1837 & 48) & ${ }^{59} \mathrm{Co}$ & 2725 \\
\hline 21) & ${ }^{39} \mathrm{~K}$ & 1925 & 49) & ${ }^{58} \mathrm{Ni}$ & 2825 \\
\hline 22) & ${ }^{40} \mathrm{~K}$ & 1928 & $50)$ & ${ }^{59} \mathrm{Ni}$ & 2828 \\
\hline 23) & ${ }^{41} \mathrm{~K}$ & 1931 & 51) & ${ }^{60} \mathrm{Ni}$ & 2831 \\
\hline 24) & ${ }^{40} \mathrm{Ca}$ & 2025 & 52) & ${ }^{61} \mathrm{Ni}$ & 2834 \\
\hline 25) & ${ }^{42} \mathrm{Ca}$ & 2031 & $53)$ & ${ }^{62} \mathrm{Ni}$ & 2837 \\
\hline 26) & ${ }^{43} \mathrm{Ca}$ & 2034 & 54) & ${ }^{64} \mathrm{Ni}$ & 2843 \\
\hline 27) & ${ }^{44} \mathrm{Ca}$ & 2037 & $55)$ & ${ }^{63} \mathrm{Cu}$ & 2925 \\
\hline 28) & ${ }^{46} \mathrm{Ca}$ & 2043 & 56) & ${ }^{65} \mathrm{Cu}$ & 2931 \\
\hline 29) & ${ }^{48} \mathrm{Ca}$ & 2049 & 57) & ${ }^{\text {nat }} \mathrm{Zn}^{*}$ & 3000 \\
\hline
\end{tabular}

${ }^{*}$ The ENDF/B-VII.0 neutron sub-library contains evaluated cross sections for elemental $\mathrm{Zn}$. We produced covariances for a full set of isotopes $64,66,67,68,70 \mathrm{Zn}$, considering that isotopic evaluations will be done in future. 
Table 4.3: List of heavy materials evaluated by BNL. The list corresponds to the neutron sub-library of the ENDF/B-VII.0 library [2].

\begin{tabular}{|llc|l|lll|}
\hline No. & Material & MAT & & No. & Material & MAT \\
\hline 1$)$ & ${ }^{175} \mathrm{Lu}$ & 7125 & & $17)$ & ${ }^{191} \mathrm{Ir}$ & 7725 \\
$2)$ & ${ }^{176} \mathrm{Lu}$ & 7128 & & $18)$ & ${ }^{193} \mathrm{Ir}$ & 7731 \\
$3)$ & ${ }^{174} \mathrm{Hf}$ & 7225 & & $19)$ & ${ }^{197} \mathrm{Au}$ & 7925 \\
$4)$ & ${ }^{176} \mathrm{Hf}$ & 7231 & & $20)$ & ${ }^{196} \mathrm{Hg}$ & 8025 \\
$5)$ & ${ }^{177} \mathrm{Hf}$ & 7234 & & $21)$ & ${ }^{198} \mathrm{Hg}$ & 8031 \\
$6)$ & ${ }^{178} \mathrm{Hf}$ & 7237 & & $22)$ & ${ }^{199} \mathrm{Hg}$ & 8034 \\
$7)$ & ${ }^{179} \mathrm{Hf}$ & 7240 & & $23)$ & ${ }^{200} \mathrm{Hg}$ & 8037 \\
$8)$ & ${ }^{180} \mathrm{Hf}$ & 7243 & & $24)$ & ${ }^{201} \mathrm{Hg}$ & 8040 \\
$9)$ & ${ }^{181} \mathrm{Ta}$ & 7328 & & $25)$ & ${ }^{202} \mathrm{Hg}$ & 8043 \\
$10)$ & ${ }^{182} \mathrm{Ta}$ & 7331 & & $26)$ & ${ }^{204} \mathrm{Hg}$ & 8049 \\
$11)$ & ${ }^{182} \mathrm{~W}$ & 7431 & & $27)$ & ${ }^{204} \mathrm{~Pb}$ & 8225 \\
$12)$ & ${ }^{183} \mathrm{~W}$ & 7434 & $28)$ & ${ }^{206} \mathrm{~Pb}$ & 8231 \\
$13)$ & ${ }^{184} \mathrm{~W}$ & 7437 & & $29)$ & ${ }^{207} \mathrm{~Pb}$ & 8234 \\
$14)$ & ${ }^{186} \mathrm{~W}$ & 7443 & $30)$ & ${ }^{208} \mathrm{~Pb}$ & 8237 \\
$15)$ & ${ }^{185} \mathrm{Re}$ & 7525 & & $31)$ & ${ }^{209} \mathrm{Bi}$ & 8325 \\
\hline
\end{tabular}




\section{Chapter 5}

\section{Conclusions and outlook}

We have applied the EMPIRE-KALMAN method to produce a simple, yet consistent set of fast neutron covariance matrices for 88 materials, 57 structural and 31 heavy nuclei, included in the ENDF/B-VII.0 library. This follows the previous work on 219 fission products materials [6] and completes the massive task of the low-fidelity project. Our results are based on model calculations and depend on the assumed uncertainties of the model parameters.

The experimental data were used only globally and approximately to ensure that calculated cross section uncertainties were not unreasonable when compared with the spread of the measurements. The same global set of model parameters and their related uncertainties was used for all 88 nuclides. The calculated cross sections and their uncertainties, often deviate from the evaluated cross sections and uncertainties derived from experimental data. This is the limitation of the global approach. In view of this, our covariances should not be associated with the ENDF/B-VII.0 library.

Our present results confirm previous notions regarding the structure of the uncertainties plotted as a function of incident energy and mass number (atomic number dependence might also be possible). In particular, we note very similar patterns observed in the total and elastic channels. A reflection of these patterns is also found in the inelastic channel. The $(n, 2 n)$ and capture channels do not seem to be affected by the structure seen in the total and elastic channels. Instead they display short range fluctuations as a function of mass number. High and low uncertainties alternately produce vertical lines on the plots. Since all nuclei were treated on the same footing using the same set of models and default set of parameters, it should be possible to explain the patterns in terms of the physics underlying our calculations. 
For example, the structure showing up in the total and elastic channels arises from the optical model and we understand the origin of deep minima in the cross section uncertainties at certain energies. Mass dependence of the energies at which these minima occur is most likely responsible for the creation of the characteristic patterns in Figs. 3.12 and 3.13.

In the future, we intend to address the intriguing structure observed in cross section uncertainties and determine its physics background.

The low-fidelity covariance project and the results that we obtained can be used as a useful starting point for any future effort in covariances. Such an effort would obviously be a step forward in using more refined modeling, e.g., local rather than global model parametrization and more explicit use of experimental data. 


\section{List of Figures}

2.1 Calculated ${ }^{89} \mathrm{Y}$ neutron cross sections for different reaction channels. . . . . . . . . . . . . . . . . . 12

2.2 Energy dependence of the sensitivity to $V_{v}^{t g}$ for the most important reaction channels in $\mathrm{n}+{ }^{89} \mathrm{Y}$ (cumulative effects). . . . 13

2.3 Energy dependence of the sensitivity to $V_{v}^{t g}$ for the most important reaction channels in $\mathrm{n}+{ }^{89} \mathrm{Y}$ (incoming channel). . . . 14

2.4 Energy dependence of the sensitivity to $V_{v}^{t g}$ for the most important reaction channels in $\mathrm{n}+{ }^{89} \mathrm{Y}$ (outgoing channel). . . . 14

2.5 Sensitivity to model parameters for ${ }^{89} \mathrm{Y}(\mathrm{n}, \gamma)$ reaction. . . . . . 15

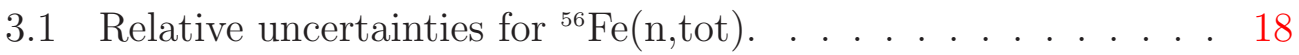

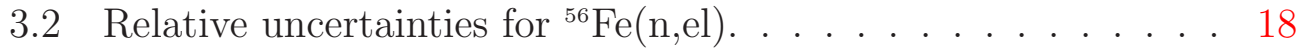

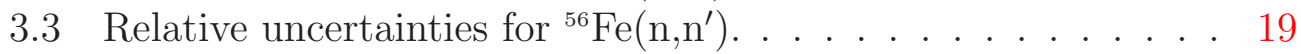

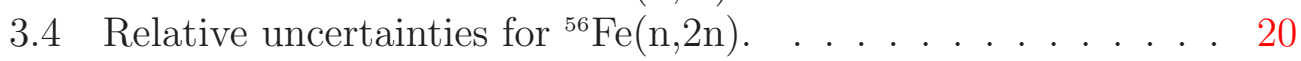

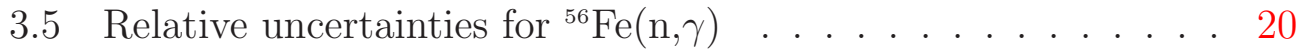

3.6 The full correlation matrix for ${ }^{56} \mathrm{Fe}(\mathrm{n}, \mathrm{tot})$ cross sections. Shown are the relative uncertainties and the $(30 \times 30)$ correlation matrix in its shortened triangular form. . . . . . . . . . . . . . 22

3.7 Relative uncertainty comparison between low-fidelity calculations and three major nuclear data libraries. . . . . . . . . . 23

3.8 Relative uncertainty comparison between low-fidelity calculations and three major nuclear data libraries. . . . . . . . . . 25

3.9 Relative uncertainty comparison between low-fidelity calculations and three major nuclear data libraries. . . . . . . . . . 25

3.10 Relative uncertainty comparison between low-fidelity calculations and three major nuclear data libraries. . . . . . . . 26

3.11 Relative uncertainty comparison between low-fidelity calculations and three major nuclear data libraries. . . . . . . . . . 26

3.12 Relative uncertainties for the total cross sections on 307 materials obtained with the EMPIRE-KALMAN method in the fast neutron energy region. . . . . . . . . . . . . . 28 
3.13 Relative uncertainties for the elastic cross sections on 307 materials obtained with the EMPIRE-KALMAN method in the fast neutron energy region. . . . . . . . . . . . . . . . . . . 29

3.14 Relative uncertainties for the inelastic cross sections on 307 materials obtained with the EMPIRE-KALMAN method in the fast neutron energy region. . . . . . . . . . . . 30

3.15 Relative uncertainties for the $(\mathrm{n}, 2 \mathrm{n})$ cross sections on 307 materials obtained with the EMPIRE-KALMAN method in the fast neutron energy region. . . . . . . . . . . . . 31

3.16 Relative uncertainties for the capture cross sections on 307 materials obtained with EMPIRE-KALMAN method in the fast neutron energy region. . . . . . . . . . . . . . 32 


\section{List of Tables}

2.1 The uncertainties used for the optical model parameters. . . . 10

2.2 The uncertainties used for nuclear level densities and preequilibrium emission. . . . . . . . . . . . . . 10

3.1 List of 307 materials evaluated by BNL. The list covers all materials in the neutron sub-library of ENDF/B-VII.0, from

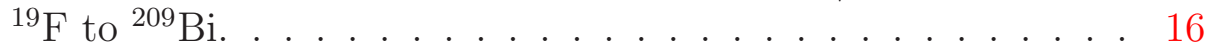

4.1 The list of 30 neutron incident energies used in the calculations of cross section covariances. . . . . . . . . . . . . . . 33

4.2 List of structural materials evaluated by BNL. . . . . . . . . . 34

4.3 List of heavy materials evaluated by BNL. . . . . . . . . . 35 


\section{Acknowledgments}

The authors would like to thank R. Little and T. Kawano (LANL), M. Dunn and M. Williams (ORNL) as well as R. McKnight (ANL) for stimulating discussions and collaboration in this project. We owe a special thanks to D.L. Smith (ANL) for his valuable comments.

The present work was supported by the DOE-NNSA within the Nuclear Criticality Safety Program and this support is gratefully acknowledged. The National Nuclear Data Center is sponsored by the Office of Nuclear Physics, Office of Science of the U.S. Department of Energy under contract No. DEAC02-98CH10886 with Brookhaven Science Associates, LLC. 


\section{Bibliography}

[1] M. Salvatores, et al. WPEC Subgroup 26: Uncertainty and Target Accuracy Assessment for Innovative Systems Using Recent Covariance Data Evaluations. Technical Report (draft), 2007.

[2] M.B. Chadwick, P. Obložinský, M. Herman, et al. ENDF/B-VII: Next Generation Evaluated Nuclear Data Library for Nuclear Science and Technology. Nuclear Data Sheets, 107(12):2931-3060, December 2006.

[3] M. Salvatores, et al. Data Adjustment for GNEP, Global Nuclear Energy Partnership. Proposal, 2007.

[4] M. Herman, R. Capote, B.V. Carlson, et al. EMPIRE: Nuclear Reaction Model Code System for Data Evaluation. Nuclear Data Sheets, 108(12):2655-2715, December 2007.

[5] T. Kawano and K. Shibata. Covariance Evaluation System [in Japanese]. Internal report JAERI-Data/Code 97-037, Japan Atomic Energy Research Institute, September 1997.

[6] M.T. Pigni, M. Herman, P. Obložinský, et al. Low-fidelity cross sections covariances for 219 fission products in the fast neutron region. Internal report BNL-79261-2007-IR, Brookhaven National Laboratory, National Nuclear Data Center, August 2007.

[7] M.T. Pigni, M. Herman, P. Obložinský, et al. Extensive set of lowfidelity covariances in fast neutron region. In $8^{\text {th }}$ International Meeting on Nuclear Applications of Accelerator Technology, Pocatello, ID, July 2007.

[8] T. Belgya, O. Bersillon, R. Capote, et al. Handbook for calculations of nuclear reaction data, Reference Input Parameter Library-2. Technical report IAEA-TECDOC-1506, International Atomic Energy Agency, Vienna, Austria, June 2006. Available at www-nds.iaea.org/RIPL-2/. 
[9] A.J. Koning and J.P. Delaroche. Local and global nucleon optical model from $1 \mathrm{keV}$ to $200 \mathrm{MeV}$. Nucl. Phys. A, 713(3-4):231-310, January 2003.

[10] J.A. Harvey. Private communication, 1987.

[11] I.A. Korzh, V.A. Mishchenko, E.N. Mozhzhukhin, et al. Differential scattering cross sections of 1.5-3.0 MeV neutrons for Ti, Fe and Bi. Ukr. Fiz. Zh., 22(1):87-94, 1977.

[12] O.A. Shcherbakov, A.I. Stupak, and A.N. Glukhovec. Slow neutron total and radiative capture cross-section of ${ }^{56} \mathrm{Fe}$. Progress Report 25, 1977. Yadernye Konstanty.

[13] B. Joensson, K. Nyberg, and I. Bergqvist. High resolution measurements of gamma rays produced by $15 \mathrm{MeV}$ neutrons. Ark. Fys., 39:295-311, January 1969.

[14] S.P Simakov, A.A Androsenko, P.A. Androsenko, et al. 14-MeV facility and research in IPPE. Vop. At. Nauki i Tekhn., Ser. Yadernye Konstanty, (4):93, March 1992.

[15] Shi Xiamin, Shen Ronglin, Xing Jinjiang, et al. Measurements of the induced gamma ray cross sections by $14.2 \mathrm{MeV}$ neutrons with $\mathrm{Fe}, \mathrm{Ni}$, Cu. Chin. J. Nucl. Phys. (Beijing), 4(2):120, 1982.

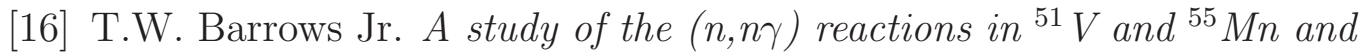
the reaction ${ }^{19} F(d, n)^{20} \mathrm{Ne}$. Ph.D. thesis or dissertation, University of Kentucky, Lexington, KY, April 1965.

[17] J. Frehaut and G. Mosinski. Measurement of the (n,2n) cross-sections for ${ }^{56} \mathrm{Fe},{ }^{59} \mathrm{Co},{ }^{89} \mathrm{Y},{ }^{169} \mathrm{Tm},{ }^{175} \mathrm{Lu},{ }^{181} \mathrm{Ta},{ }^{197} \mathrm{Au},{ }^{209} \mathrm{Bi},{ }^{238} \mathrm{U}$ and of the $(\mathrm{n}, 3 \mathrm{n})$ cross-section for ${ }^{238} \mathrm{U}$ from threshold to $15 \mathrm{MeV}$ incident neutron energy. Technical report CEA-R-4627, Centre d'Études Nucléaires, Saclay, Gifsur-Yvette, France, 1974.

[18] V. Corcalciuc, B. Holmqvist, A. Marcinkowski, et al. A study of the neutron induced reactions for ${ }^{19} \mathrm{~F},{ }^{56} \mathrm{Fe},{ }^{59} \mathrm{Co}$ in the energy interval 16 to $22 \mathrm{MeV}$. Nucl. Phys. A, 307(3):445-471, September 1978.

[19] S.M. Qaim and N.I. Molla. A systematic study of $(\mathrm{n}, \mathrm{p})$ reactions at 14.7 MeV. Nucl. Phys. A, 283(2):269-288, June 1977.

[20] R.L. Macklin, P.J. Pasma, and J.H. Gibbons. Resonance Neutron Capture and Transmission in Sulfur, Iron, and Lead. Phys. Rev., 136(3B):B695-B702, November 1964. 
[21] A. Ernst, F.H. Fröhner, and D. Kompe. High resolution measurements of radiative neutron capture in ${ }^{47} \mathrm{Ti},{ }^{56} \mathrm{Fe},{ }^{58} \mathrm{Ni},{ }^{60} \mathrm{Ni}$ and ${ }^{61} \mathrm{Ni}$ between 7 and $200 \mathrm{keV}$. In $2^{\text {nd }}$ International Conference on Nuclear Data for Reactors, volume 1, page 633, Helsinky, Finland, June 1970.

[22] Huang Zheng-De, Cao Zhong, Wang Hui-Zhu, et al. Radiative capture of $14.2 \mathrm{MeV}$ neutron by ${ }^{56} \mathrm{Fe}$ and ${ }^{238} \mathrm{U}$. Conference Report LBL-11118, Lawrence Berkeley Laboratory, 1980. 